\title{
REPRESENTATIONS OF GROUPS AS QUOTIENT GROUPS. II. MINIMAL CENTRAL CHAINS OF A GROUP
}

\author{
BY \\ REINHOLD BAER
}

The descending chain of normal subgroups $N_{0}, N_{1}, \cdots, N_{i}, \cdots$ of the group $G$ is said to be a central chain (1) of $G$ if $N_{i-1} / N_{i}$ is, for positive $i$, part of the center of $G / N_{i}$. This is equivalent to the requirement

$$
\left(G, N_{i-1}\right) \leqq N_{i} \quad \text { for } 0<i,
$$

if we define $(U, V)$, for $U$ and $V$ subgroups of $G$, as the subgroup generated by all the commutators $(u, v)=u^{-1} v^{-1} u v$ for $u$ in $U$ and $v$ in $V$. A central chain is clearly minimal if we substitute equality for the inequality just mentioned. It is obvious that a minimal central chain is completely determined by its first member, and that every normal subgroup $N$ of $G$ is the first term of the minimal central chain ${ }_{i} N$, defined inductively as follows:

$$
{ }_{0} N=N, \quad{ }_{i} N=\left(G,{ }_{i-1} N\right)
$$

for positive $i$.

We shall refer to this chain ${ }_{i} N$ as the minimal central chain of $G$, determined by $N$.

A special example is the well known lower central chain of $G$ which we denote by ${ }^{i} G$; it is in our terminology the minimal central chain of $G$, determined by $G$. Every central chain $N_{i}$ is related to the lower central chain of $G$ by the following important inequality:

$$
\left({ }^{i} G, N_{j}\right) \leqq N_{i+j+1}
$$

It is furthermore easily deduced by complete induction from (2) that

$$
{ }_{i} N \leqq i G \quad \text { for every } N \text { and every } i \text {. }
$$

It is clear that ${ }_{i-1} N /{ }_{i} N$ is part of the center of $G /{ }_{i} N$, and thus $G /{ }_{i} N$ is a so-called central extension of ${ }_{i-1} N /{ }_{i} N$ by $G /{ }_{i-1} N$. Thus the theory of central extensions will be of paramount importance for this investigation. It will be necessary to introduce new concepts into this theory and to obtain new results, and some of these seem to be of interest independent of the objects for which they are introduced here.

Presented to the Society, September 17, 1945; received by the editors November 20,1944.

(1) This concept is due to P. Hall [1]. Numbers in brackets refer to the Bibliography at the end of the paper.

(2) For a proof of this inequality see, for example, Zassenhaus [1, p. 120]. 
The two fundamental results of this investigation may be stated shortly as follows: If $G / N$ is a finite group, then ${ }^{i} G /{ }_{i} N$ is finite for every positive $i$; if $N$ is a normal subgroup of the free group $G$, then the manifold of all the groups ${ }^{i} G / W$ such that ${ }_{i} N /{ }_{i+1} N$ is the direct product of $W /{ }_{i+1} N$ and $\left({ }_{i} N \cap{ }^{i+1} G\right) /{ }_{i+1} N$ is (in a certain sense) a homomorphic image of the manifold of all the abelian extensions of $\left({ }_{i} N \cap{ }^{i+1} G\right) /{ }_{i+1} N$ by ${ }^{i} G /\left({ }_{i} N{ }^{i+1} G\right)$. The importance of these results stems from the fact that, for free $G$, the groups ${ }^{i} G /{ }_{i} N,{ }^{i} G /\left({ }_{i} N{ }^{i+1} G\right)$ and $\left({ }_{i} N \cap{ }^{i+1} G\right) /{ }_{i+1} N$ are known $\left({ }^{3}\right)$ to be invariants of the group $G / N$; and that the groups ${ }^{i} G / W$ may be considered as generalizations of I. Schur's "Darstellungsgruppen." Though superficially these two results may look rather disconnected, the means of proof are very similar, and in particular some criteria on the "splitting of central extensions" will be powerful tools for both proofs.

We have written this second part of our investigation concerning the representations of groups as quotient groups in such a way that it may be understood without any knowledge of the first part. In this first part we have introduced the lower central series of a group $G$ relative to a normal subgroup $N$ of $G$, and this concept is identical with the present concept of "minimal central chain." We have given only a rather sketchy discussion of this concept in the first part, and the present investigation may help to fill this gap.

1. Central factor sets and the structure of $N /(G, N)$. The study of $N /(G, N)$ is closely connected with the investigation of central extensions. Thus we recall the main concepts of the latter theory $\left({ }^{4}\right)$. The group $G$ is said to be a central extension of the group $Z$ by the group $E$ if $Z$ is part of the center of $G$ and if $G / Z$ is isomorphic to the group $E$. Denote by $r(x)$ a representative of the coset of $G / Z$ which corresponds to the element $x$ in $E$, but let $r(1)=1$. Then the elements $f(x, y)=r(x) r(y) r(x y)^{-1}$ for $x, y$ in $E$ belong to $Z$, and they satisfy the relations:

$$
f(x, 1)=f(1, y)=1, \quad f(x, y) f(x y, z)=f(y, z) f(x, y z) .
$$

This function $f(x, y)$ is a central factor set of $E$ in the abelian group $Z$ which is realized by the extension $G$ of $Z$ by $E$, and the central extension $G$ of $Z$ by $E$ is completely determined by this central factor set. We note furthermore that every function satisfying (5) is termed a central factor set of $E$ in $Z$, and that every central factor set of $E$ in $Z$ may be realized by one and essentially only one central extension of $Z$ by $E$.

Assume now that the representatives $r(x)$ of the cosets of $G / Z$ have been selected in such a way that $G$ is generated by them $\left.{ }^{5}\right)$. Then there exist, to every element $z$ in $Z$, elements $x_{1}, \cdots, x_{n}$ in $E$ such that $z=r\left(x_{1}\right) \cdots r\left(x_{n}\right)$. It follows readily from the definition of the elements $f(x, y)$ that

(3) For a sketch of a proof see Baer [4] (or Part I of this investigation).

(4) For details of this theory, see, for example, Zassenhaus [1].

(5) This hypothesis is not always satisfied. A simple example is $G=Z \neq 1$. 
$z=z^{\prime} r\left(x_{1} \cdots x_{n}\right)=z^{\prime} r(e)$ where $z^{\prime}$ is a product of suitable elements $f(u, v)$. But $z$ and $z^{\prime}$ are both in $Z$. Hence $r(e)$ is the representative of $Z$, proving that $e=1$ and that therefore $r(e)=1$ or $z=z^{\prime}$. Thus we have shown that $Z$ is generated by the elements $f(x, y)$ if $G$ is generated by the representatives $r(x)$.

Noting now that $G /(N, G)$ is a central extension of $N /(N, G)$ by $G / N$, we obtain the following fact.

THEOREM 1. If $N$ is a normal subgroup of the group $G$, and if there exists a set of representatives of the cosets, not $N$, of $G / N$ which generates $G /(N, G)$, then the central extension $G /(N, G)$ of $N /(N, G)$ by $G / N$ is determined by a central factor set which generates $N /(N, G)$.

Remark 1. It is impossible to omit the hypothesis of the existence of a set of representatives of $G / N$ which generates $G /(N, G)$. This may be seen from the following general considerations. Let $M$ be a normal subgroup of a group $H$, and let $K$ be some group. Denote by $G$ the direct product of $H$ and $K$, and by $N$ the direct product of $M$ and $K$. Then $G / N$ and $H / M$ are isomorphic groups, and $(G, N)$ is the direct product of $(H, M)$ and $(K, K)$, so that $N /(G, N)$ is the direct product of $M /(H, M)$ and $K /(K, K)$. But the nature of this second factor is completely independent of the structure of $G / N$.

To show that the assertion of Theorem 1 cannot be improved, we consider the free central factor set of a group $E$. To define this denote by $E^{*}$ the abelian group generated by elements $f(x, y)$ for $x, y$ in $E$, subject to the relations (5). The elements $f(x, y)$ then form the desired free central factor set.

THEOREM 2. If $N$ is a normal subgroup of the free group $G$, and if there exists a set of representatives of the cosets, not $N$, of $G / N$ which is at the same time a free set of generators of $G$, then $N /(G, N)$ is generated by the free central factor set of $G / N$, and this free central factor set is realized by the central extension $G /(G, N)$ of $N /(G, N)$ by $G / N$.

Proof. If $E=G / N$, then there exists a central extension $G^{*}$ of $E^{*}$ by $E$ which realizes the free central factor set $f(x, y)$ of $E$ in $E^{*}$. Hence there exists a set of representatives $r^{*}(x)$ such that $f(x, y)=r^{*}(x) r^{*}(y) r^{*}(x y)^{-1}$ for $x, y$ in $E$, and $r^{*}(1)=1$. There exists furthermore a set of representatives $r(x)$ of the cosets of $G / N$ such that the elements $r(x)$ for $x \neq 1$ form a free set of generators of the free group $G$. There exists finally one and only one homomorphism of $G$ upon $G^{*}$ by which $r(x)$ is mapped upon $r^{*}(x)$. This homomorphism maps $(G, N)$ upon 1 and $N$ upon $E^{*}$, since it maps, by Theorem 1 , a factor set generating $N /(G, N)$ just upon the elements $f(x, y)$. Thus it follows that $(N, G)$ is the kernel $\left(^{6}\right)$ of this homomorphism, and now our contention is readily verified.

Remark 2. Every group may be represented in the form $G / N$ for $G$ a free

(6) The kernel of a homomorphism consists of the elements mapped upon 1 by this homomorphism. 
group possessing a free set of generators which is at the same time a set of representatives of the cosets, not $N$, of $G / N$. Thus Theorem 2 (or the existence of the central extension $G^{*}$ of $E^{*}$ by $E$ ) shows the impossibility of improving Theorem 1.

If $J$ is the additive group of real numbers modulo 1 , then the characters of an abelian group are just its homomorphisms into $J$.

COROLlARY. The character group of $E^{*}$ and the group of central factor sets of $E$ in $J$ are essentially the same.

Proof. If $f(x, y)$ is the free central factor set of the group $E$ in $E^{*}$, then $f(x, y)$ generates $E^{*}$. Thus a character of $E^{*}$ is 0 if, and only if, it maps $f(x, y)$ upon 1. It is an immediate consequence of the definition of $E^{*}$ and of a factor set that every character maps $f(x, y)$ upon a central factor set of $E$ in $J$, and from a previous remark we deduce that different characters map $f(x, y)$ upon different factor sets. It is an immediate consequence of the definition of $E^{*}$ that every factor set of $E$ in $J$ is obtained in this fashion, and this completes the proof.

This theorem is a counterpart to a theorem of Eilenberg-MacLane [1, 2]; see Remark 2 of $\$ 2$ below. For an interesting improvement upon the above result cp. Corollary 2 of $\$ 3$ below.

2. Central transformation sets and the structure of $[(G, G) \cap N] /(G, N)$. If $t(x)$ is, for every element $x$ in the group $E$, an element in the abelian group $Z$, and if $t(1)=1$, then $t(x, y)=t(x) t(y) t(x y)^{-1}$ is easily seen to be a central factor set of $E$ in $Z$. Every central factor set that may be represented in this fashion is termed a central transformation set of $E$ in $Z$. If $G$ is the central extension of $Z$ by $E$ which is determined by the central factor set $f(x, y)$, then a necessary and sufficient condition for $Z$ to be a direct factor of $G$ is that $f(x, y)$ be a central transformation set of $E$ in $Z$. Two central factor sets $a(x, y)$ and $b(x, y)$ of $E$ in $Z$ determine essentially the same central extension of $Z$ by $E$ if, and only if, $a(x, y) b(x, y)^{-1}$ is a central transformation set of $E$ in $Z$, and any two central factor sets meeting this requirement are termed associate central factor sets $\left({ }^{7}\right)$.

LEMMA 1. If the group $G$ is a central extension of the group $Z$ by the group $E$, and if the subgroup $R$ of $G$ is generated by a set of representatives $r(x)$ of the cosets of $G / Z(r(1)=1)$, then:

(i) $R \cap Z$ is generated by the factor set $r(x) r(y) r(x y)^{-1}$.

(ii) $(G, G)=(R, R)$.

(iii) $(G, G) \cap Z \leqq R \cap Z$.

Proof. (i) is a consequence of Theorem 1 of $\$ 1$, since $R$ is a central extension of $R \cap Z$ by $E$ which is generated by a set of representatives of the cosets of $R /(R \cap Z)$. If $g, h$ are elements in $G$, then there exist elements $g^{\prime}, h^{\prime}$ in $Z$

(7) See footnote 4. 
and elements $g^{\prime \prime}, h^{\prime \prime}$ in $E$ such that $g=g^{\prime} r\left(g^{\prime \prime}\right)$ and $h=h^{\prime} r\left(h^{\prime \prime}\right)$. Since $Z$ is part of the center of $G$, we have clearly $(g, h)=\left(r\left(g^{\prime \prime}\right), r\left(h^{\prime \prime}\right)\right)$. This proves (ii), and (iii) is an immediate consequence of (ii).

If $H$ and $K$ are central extensions of the groups $U$ and $V$ respectively by the same group $E$, then the homomorphism $\eta$ of $H$ into $K$ is said to induce the identity in $E$, if the cosets $U x$ and $V x^{n}$ correspond, for every $x$ in $H$, to the same element in $E$.

Lemma 2. If $G$ is a central extension of the group $Z$ by the group $E$, and if $\eta$ is a homomorphism of the group $Z$ into the abelian group $Y$, then there exists a homomorphism of $G$ into an essentially uniquely determined central extension $F$ of $Y$ by $E$ which induces $\eta$ in $Z$ and the identity in $E$.

Proof. If $r(x)$ is, for every $x$ in $E$, an element in the coset of $G / Z$ which corresponds to the element $x$ in $E$, and if $r(1)=1$, then the central extension $G$ of $Z$ by $E$ is determined by the central factor set $f(x, y)=r(x) r(y) r(x y)^{-1}$ of $E$ in $Z$. Clearly $f(x, y) \eta$ is a central factor set of $E$ in $Y$, and it determines uniquely a central extension $F$ of $Y$ by $E$. It is possible to select in the coset of $F / Y$ which corresponds to the element $x$ in $E$ an element $t(x)$ such that $t(1)=1$ and $f(x, y)^{n}=t(x) t(y) t(x y)^{-1}$. Clearly there exists one and only one homomorphism $\kappa$ of $G$ into $F$ which maps $r(x)$ upon $t(x)$ and which induces $\eta$ in $Z$. Now it is obvious how to complete the proof.

THEOREM 1. If $G$ is the central extension of the group $Z$ by the group $E$ which is determined by the central factor set $f(x, y)$ of $E$ in $Z$, then the following properties of the subgroup $S$ of $Z$ imply each other.

(i) $(G, G) \cap Z \leqq S$.

(ii) There exists an abelian group $T$ which contains $Z / S$ such that $S f(x, y)$ is a central transformation set of $E$ in $T$.

Proof. From our hypothesis we infer the existence of an element $r(x)$ in the coset of $G / Z$ which corresponds to the element $x$ in $E$ such that $r(1)=1$ and $f(x, y)=r(x) r(y) r(x y)^{-1}$.

If (i) holds true, then $S(G, G) \cap Z=S((G, G) \cap Z)=S$. Hence $T=G /(G, G) S$ is an abelian group whose subgroup $Z(G, G) S /(G, G) S$ is essentially the same as $Z /(Z \cap(G, G) S)=Z / S$. If $t(x)=(G, G) S r(x), t(x) t(y) t(x y)^{-1}=(G, G) S f(x, y)$, and now it is evident that (ii) is a consequence of (i).

If (ii) holds true, then denote by $\eta$ the homomorphism of $Z$ into $T$ which maps the element $z$ in $Z$ upon the element $S z$ in $T$. We deduce from Lemma 2 the existence of a central extension $F$ of $T$ by $E$ and the existence of a homomorphism $\phi$ of $G$ into $F$ which induces $\eta$ in $Z$ and which maps $r(x)$ upon an element in the coset of $F / T$ which corresponds to the element $x$ in $E$. From (ii) we infer now that $f(x, y)^{\phi}=f(x, y)^{\eta}$ is a central transformation set of $E$ in $T$. Since $f(x, y)^{\phi}$ is a central factor set of $E$ in $T$ which determines the central extension $F$ of $T$ by $E$, it follows now that $T$ is a direct factor of $F$. Conse- 
quently we deduce from the commutativity of $T$ or from Lemma 1 that $(F, F) \cap T=1$. But $(G, G)^{\phi} \leqq(F, F)$ and $Z^{\phi} \leqq T$ imply now that $((G, G) \cap Z)^{n}$ $\leqq(G, G)^{\phi} \cap Z^{\phi} \leqq(F, F) \cap T=1$. Consequently $(G, G) \cap Z$ is part of the kernel $S$ of $\eta$, as we desired to prove.

CoRollary $\left.1{ }^{8}\right)$. If $G$ is the central extension of the group $Z$ by the group $E$ which is determined by the central factor set $f(x, y)$ of $E$ in $Z$, then the following properties of the homomorphism $\eta$ of $Z$ into the abelian group $A$ imply each other.

(i) $((G, G) \cap Z)^{\eta}=1$.

(ii) There exists an abelian group $T$ which contains $A$ such that $f(x, y)^{\eta}$ is a central transformation set of $E$ in $T$.

Proof. We denote by $S$ the kernel of the homomorphism $\eta$ of $Z$. Since the subgroup $Z^{\eta}$ of $A$ and the quotient group $Z / S$ are isomorphic groups, we may deduce from condition (i) and from Theorem 1 the existence of an abelian group $B$ which contains $Z^{\eta}$ such that $f(x, y)^{\eta}$ is a central transformation set of $E$ in $B$. We may consider $A$ and $B$ subgroups of an abelian group $T$ such that $A B=T$ and $A \cap B=Z^{\eta}$, and this shows that (ii) is a consequence of (i). That (i) is conversely a consequence of (ii) is an immediate consequence of Theorem 1 applied to the kernel $S$ of $\eta$.

Remark 1. It is clearly impossible to deduce from (i) of Corollary 1 the fact that $f(x, y)^{\eta}$ is a central transformation set of $E$ in $A$, since an abelian extension of the group $A$ by an abelian group need not contain $A$ as a direct factor.

Remark 2. If we apply this Corollary 1 upon the corollary of $\S 1$, then we see that the characters of $Z$ which map $(G, G) \cap Z$ upon 1 are just those characters which map $f(x, y)$ upon a central transformation set of $E$ in $J$. Thus we obtain the connection between $(N \cap(H, H)) /(H, N)$ and the group of central extensions of $J$ by $H / N$, for $N$ a normal subgroup of the group $I$, which has been established by Eilenberg-MacLane $[1,2]$.

THEOREM 2. If $N$ is a normal subgroup of the group $G$, and if $G /(G, G)$ is a free abelian group, then $N /[N \cap(G, G)]$ is a free abelian group, and $[N \cap(G, G)] /(N, G)$ is a direct factor of $N /(N, G)$.

Proof. The group $N /[N \cap(G, G)]$ is isomorphic to the subgroup $N(G, G) /(G, G)$ of $G /(G, G)$. The latter group is a free abelian group, and the subgroups of free abelian groups are known to be free abelian groups $\left({ }^{9}\right)$. This proves our first assertion of which the sccond one is an immediate consequence.

Lemma 3. Suppose that $M$ and $N$ are normal subgroups of the group $G$. Then $M N / M$ is a direct factor of $G / M$ if, and only if, there exists a normal subgroup $S$ of $G$ such that $M \leqq S$ and $N \cap M=N \cap S, S N=G$.

(8) Theorem 2 of $\$ 6$ constitutes an essential generalization of this result.

(9) Proofs of this result may be found in Baer [2] or Lefschet\% [1]. 
Proof. $M N / M$ is a direct factor of $G / M$ if, and only if, there exists a normal subgroup $S$ of $G$ which contains $M$ and which satisfies $S N=G$ and $S \cap M N=M$. But $S \cap M N=M(S \cap N)$, since $M \leqq S$. Thus $S \cap N \leqq M$ is a necessary and sufficient condition for $M=S \cap M N$. From $M \leqq S$ we deduce finally that $S \cap N=M \cap N$ is equivalent to $S \cap N \leqq M$, and this completes the proof.

TheOREM 3. If $N$ is a normal subgroup of the group $G$, and if $N /(N, G)$ is the direct product of $[(G, G) \cap N] /(N, G)$ and the subgroup $S$, then the following conditions imply each other.

(i) $S$ is a direct factor of $G /(N, G)$.

(ii) $N(G, G) /(G, G)$ is a direct factor of $G /(G, G)$.

Proof. We note first that $N(G, G) /(N, G)$ is the direct product of $S$ and of $(G, G) /(N, G)$. If (i) is satisfied by $S$, then $G /(N, G)$ is the direct product of $S$ and of a subgroup $T$. Since the cross cut of $S$ and $(G, G) /(N, G)$ is 1 , it follows that $T$ contains $(G, G) /(N, G)$. The cross cut of $T$ and $N(G, G) /(N, G)$ is therefore $(G, G) /(N, G)$. Hence $G /(G, G)$ is the direct product of $N(G, G) /(G, G)$ and of $T /[(G, G) /(N, G)]$.

Suppose conversely that (ii) is satisfied by $G$ and $N$. Then we deduce from Lemma 3 the existence of a normal subgroup $V$ of $G /(N, G)$ which contains $(G, G) /(N, G)$, satisfies $V[N /(N, G)]=G /(N, G)$ and whose cross cut with $N /(N, G)$ equals the cross cut of $N /(N, G)$ and of $(G, G) /(N, G)$. But the latter cross cut is equal to $[(G, G) \cap N] /(N, G)$. This implies in particular that $S \cap V=1$. Since $V$ contains $(G, G) /(N, G)$, and since $N /(N, G)$ is the product of $S$ and $[N \cap(G, G)] /(N, G)$, we infer that $S V$ contains $N /(N, G)$, proving that $G /(N, G)$ is the direct product of $S$ and $V$.

Corollary 2. If $N$ is a normal subgroup of $G$, and if $G /(G, G)$ is a free abelian group, then the following two properties of $N$ imply each other.

(i) $G /(N, G)$ is the direct product of a subgroup $S$ of $N /(N, G)$ and of a group $T$ satisfying $T \cap[N /(N, G)]=[N \cap(G, G)] /(N, G)$.

(ii) $G / N(G, G)$ is a free abelian group.

Proof. It is a consequence of Theorem 2 that $N /(N, G)$ is the direct product of $[N \cap(G, G)] /(N, G)$ and of a subgroup $S$. If furthermore condition (ii) is satisfied by $N$, then $N(G, G) /(G, G)$ is obviously a direct factor of $G /(G, G)$, and we deduce from Theorem 3 that $G /(N, G)$ is the direct product of $S$ and of a subgroup $T$. Since 1 is the cross cut of $S$ and of $(G, G) /(N, G)$, it follows that $(G, G) /(N, G)$ is part of $T$, and now it is clear that $S$ and $T$ meet the requirements of condition (i).

Suppose conversely that (i) is satisfied by $N$. Then $T$ contains $(G, G) /(N, G)$, since the cross cut of $S$ and $(G, G) /(N, G)$ is 1 . Hence $G /(G, G)$ is the direct product of $T /[(G, G) /(N, G)]$ and of $N(G, G) /(N, G)$, if we remember that $N(G, G) /(N, G)$ is the direct product of $S$ and $(G, G) /(N, G)$. Thus $G / N(G, G)$ 
is isomorphic to a subgroup of $G /(G, G)$. But $G /(G, G)$ is a free abelian group and subgroups of free abelian groups are known $\left({ }^{10}\right)$ to be free abelian groups. Hence (ii) is a consequence of (i).

Remark 3. Condition (ii) is satisfied whenever $G$ is a free group and $N \leqq(G, G)$. Thus our Corollary 2 can be applied when considering the subgroups ${ }_{i} N$ for $0<i$ and $N$ a normal subgroup of a free group. See the theorem of $\$ 5$ below.

Remark 4. The following special case of Corollary 2 is of some interest, though a direct proof of it would be rather simple. Suppose that the group $G / N$ is equal to its commutator subgroup. Then $N(G, G)=G$ so that condition (ii) is certainly satisfied, showing that $G /(N, G)$ is the direct product of $(G, G) /(N, G)$ and of a subgroup $S$ of $N /(N, G)$, provided $G /(G, G)$ is a free abelian group.

3. The free central extension. If $E^{*}$ is the abelian group which is generated by the free central factor set of the group $E$, then there exists one and essentially only one central extension $F$ of the group $E^{*}$ by the group $E$ which realizes the free central factor set of $E$ in $E^{*}$. This extension $F$ of $E^{*}$ by $E$ shall be termed the free central extension by the group $E$. It is an immediate consequence of Theorem 2 in $\S 1$ that $G /(N, G)$ is the free central extension of the group $G / N$ if $N$ is a normal subgroup of the free group $G$, and if there exists a set of representatives of the cosets, not $N$, of $G / N$ which is at the same time a free set of generators of $G$. Since in this case $G /(G, G)$ is a free abelian group, we deduce from Theorem 2 of $\$ 2$ the following fact.

THEOREM 1. If $E^{*}$ is generated by the free central factor set of the group $E$, and if $F$ is the free central extension (of the group $E^{*}$ ) by the group $E$, then $E^{*}$ is the direct product of $E^{*} \cap(F, F)$ and of a free abelian group.

If $S$ is a subgroup of $E^{*}$ such that $E^{*}$ is the direct product of $S$ and $E^{*} \cap(F, F)$, then $S$ is a normal subgroup of $F$, and we shall term $F / S$ a reduced free central extension by $E$. It is not difficult to deduce from Corollary 2 of $\$ 2$ the fact that there exists essentially only one reduced free central extension by $E$, if $E /(E, E)$ is a free abelian group. In general, however, no such uniqueness theorem is true. For a survey of the types of reduced free central extensions, see $\$ 7$ below.

Corollary 1. If $H$ is a central extension of the group $Z$ by the group $E$, then the following properties of $H$ imply each other.

(a) $Z \leqq(H, H)$.

(b) There exists a homomorphism of a suitable reduced free central extension by $E$ upon $H$ which induces the identity in $E$.

Proof. If $Z \leqq(H, H)$, then it follows from Lemma 1 of $\S 2$ that every set of representatives of the cosets, not $Z$, of $H / Z$ generates $H$. Consequently

(10) See footnote 9. 
there exists a homomorphism $\eta$ of the free central extension $F$ by $E$ upon $H$ which induces the identity in $E$ and which maps $E^{*}$ upon $Z$. (This may either be verified directly, or it may be deduced from Theorem 1 of $\S 1$ and Lemma 2 of §2.) The kernel $K$ of $\eta$ is clearly a subgroup of $E^{*}$. From $H=F^{\eta}$ we deduce $E^{* \eta}=Z \leqq(H, H)=(F, F) \eta$, and this implies $Z=\left((F, F) \cap E^{*}\right) \eta$, since $E^{*}$ is the inverse image of $Z$ under $\eta$; and since the subgroup $K$ of $E^{*}$ is the kernel of $\eta$, it follows that $E^{*}=\left((F, F) \cap E^{*}\right) K$. It is a consequence of Theorem 1 that $E^{*} /\left((F, F) \cap E^{*}\right)=\left((F, F) \cap E^{*}\right) K /\left((F, F) \cap E^{*}\right)$ is a free abelian group. But this group is isomorphic to $K /\left(K \cap(F, F) \cap E^{*}\right)=K /(K \cap(F, F))$. This implies that $K$ is the direct product of $K \cap(F, F)$ and of a free abelian group $S$, and $E^{*}$ is therefore the direct product of $(F, F) \cap E^{*}$ and of the free abelian subgroup $S$ of $K$. Clearly $F / S$ is a reduced free central extension by $E$, and the homomorphism of $F / S$ upon $H$ which consists in the mapping of $S x$ upon $(S x)^{\eta}=x^{\eta}$ meets all the requirements of (b), showing that (b) is a consequence of (a).

If $T$ is a subgroup of $E^{*}$ such that $E^{*}$ is the direct product of $T$ and $(F, F) \cap E^{*}$, then the commutator subgroup of the reduced free central extension $F / T$ is $(F, F) T / T$, and this group certainly contains $E^{*} / T$. Hence (a) is satisfied by the reduced free central extensions, and from this fact one infers immediately that (a) is a consequence of (b).

Remark 1. I. Schur $\left.{ }^{11}\right)$ has connected with every finite group $E$ certain groups which he terms "Darstellungsgruppen" of $E$. It is an immediate consequence of Corollary 1 that Schur's concept coincides with our concept of a reduced free central extension by $E$. Thus it follows from one of Schur's results that the reduced free central extensions are not uniquely determined by $E$. On the other hand he had already shown that both $(F, F)$ and $E^{*} \cap(F, F)$ are invariants of the group $E$. The latter of these invariants he had called the "Multiplikator" of $E\left({ }^{12}\right)$.

COROLLARY 2. The group of central transformation sets of $E$ in $J$ is a direct factor of the group of central factor sets of $E$ in $J$.

Proof. It is a consequence of the corollary of $\$ 1$ that the group of central factor sets of $E$ in $J$ is essentially the same as the character group of $E^{*}$. It is a consequence of Theorem 1 that $E^{*}$ is the direct product of a free abelian group $S$ and of $(F, F) \cap E^{*}$. Denote by $L^{\prime}$ the group of those characters of $E^{*}$ which annihilate $S$ and by $L^{\prime \prime}$ the group of those characters of $E^{*}$ which annihilate $(F, F) \cap E^{*}$. Then the character group of $E^{*}$ is the direct product of $L^{\prime}$ and $L^{\prime \prime}$. It is a consequence of Corollary 1 of $\$ 2$ that $L^{\prime \prime}$ consists of exactly those characters of $E^{*}$ which map the free central factor set of $E$ upon a

(11) Asana-Shoda [1], Miller [1, 2] and I. Schur [1, 2].

(12) Thus the invariance theorem of Schur is equivalent, at least for finite groups, to the invariance theorem of Hopf [1]. From a different point of view the multiplicator has been investigated by Clifford-MacLane [1]. 
central transformation set of $E$ in $J$. Thus $L^{\prime \prime}$ is essentially the same as the group of central transformation sets of $E$ in $J$, and this group is therefore a direct factor of the group of central factor sets of $E$ in $J$.

THEOREM 2. The following three properties of a group $E$, the group $E^{*}$ which is generated by the free central factor set of $E$, and the free central extension $F$ of $E^{*}$ by E imply each other.

(a) $E$ is finite.

(b) $E^{*}$ is generated by a finite number of elements.

(c) $E^{*} /\left(E^{*} \cap(F, F)\right)$ is generated by a finite number of elements.

Proof. It is obvious that (a) implies (b) and that (b) implies (c). Suppose finally that $E$ be infinite. Then we construct the free central transformation set of $E$ as follows: Denote by $T$ a free abelian group whose rank equals the number of elements, not 1 , in $E$. Then there exists a 1:1 map $t(x)$ of the elements $x \neq 1$ in $E$ upon a basis of $T$. Put $t(1)=1$, and let $t(x, y)=t(x) t(y) t(x y)^{-1}$ for $x, y$ in $E$. Then $t(x, y)$ is the desired free central transformation set of $E$. There exists clearly a homomorphism $\eta$ of $E^{*}$ into $T$ which maps the free central factor set of $E$ upon $t(x, y)$. It is a consequence of Corollary 1 of $\$ 2$ that $\left(E^{*} \cap(F, F)\right)^{\eta}=1$. Consider the elements $t\left(x, x^{-1}\right)=t(x) t\left(x^{-1}\right)$. Clearly $t\left(x, x^{-1}\right)=t\left(x^{-1}, x\right)$. But the different ones among these elements $t\left(x, x^{-1}\right)$ are readily seen to form an infinite independent subset (of elements of order 0 ) of $T$. Thus $E^{* \eta}=\left[E^{*} /\left(E^{*} \cap(F, F)\right)\right]^{\eta}$ cannot be generated by a finite number of elements if $E$ is infinite, and this shows that (a) is a consequence of (c).

THEOREM 3. If $N$ is a normal subgroup of finite index $n$ of $G$, then $((G, G) \cap N) /(N, G)$ is a finite group the orders of whose elements are divisors of $n$.

Proof. If $E=G / N$, then it is readily deduced from Lemma 1 of $\$ 2$ that $((G, G) \cap N) /(G, N)$ is a homomorphic image of $E^{*} \cap(F, F)$ where $F$ is the free central extension by $E$. Map every element in $E^{*}$ upon its $n$th power. It is well known $\left({ }^{13}\right)$ that this endomorphism maps the free central factor set of $E$ upon a central transformation set in $E^{*}$. Hence we deduce from Corollary 1 of $\S 2$ that $\left(E^{*} \cap(F, F)\right)^{n}=1$. We infer from Theorem 2 that $E^{*}$ and therefore $E^{*} \cap(F, F)$ are abelian groups which are generated by a finite number of elements, and Theorem 3 is an immediate consequence of these facts.

Remark 2. It has been pointed out in Remark 1 that $E^{*} \cap(F, F)$ is I. Schur's multiplicator of the group $E$ whose finiteness, for finite $E$, has been shown by I. Schur. A considerable extension of Theorem 3 will be given in $\$ 6$ below. It should be noted that the converse statement is false, since the multiplicator of an infinite cyclic group is 1 .

Corollary 3. If $E$ is finite, then $E^{*} \cap(F, F)$ is the subgroup of the elements of finite order in $E^{*}$.

(13) For a proof, see Zassenhaus [1, Satz 24, p. 125]. 
This is an immediate consequence of Theorems 1 and 3.

Corollary 4. The quotient groups $G /{ }_{i} N$, for $N$ a normal subgroup of $G$, are all finite if, and only if, $G / N$ and $N(G, G) /(G, G)$ are finite.

Proof. Since $N(G, G) /(G, G)$ is isomorphic to $N /(N \cap(G, G))$, it is a homomorphic image of the subgroup $N /(N, G)$ of $G /{ }_{1} N$. This shows the necessity of the second condition whereas the necessity of the first condition is a consequence of $G /{ }_{0} N=G / N$.

Assume now that the conditions are satisfied. Then $G /{ }_{0} N$ is finite; and thus we may make the induction hypothesis that $G /{ }_{i-1} N$, for positive $i$, be finite. Let $M={ }_{i-1} N$. Then $(M, G)={ }_{i} N$. Since $G / M$ is finite, we deduce from Theorem 3 the finiteness of $((G, G) \cap M) /(M, G)$. If $1<i$, then $M \leqq(G, G)$ or $M=(G, G) \cap M$, proving the finiteness of $M /(M, G)$. If $i=1$, then $M=N$, and $M /(M \cap(G, G))=N /(N \cap(G, G))$ is finite since it is isomorphic to the group $N(G, G) / N$ which is finite by hypothesis. Hence $M /(M, G)$ is finite in every case. From our induction hypothesis we deduce the finiteness of $G / M$, and thus we have shown that $G /(M, G)=G /{ }_{i} N$ is finite, as we desired to show.

Remark 3. If $G$ is the direct product of a finite group $E$ and an infinite abelian group $N$, then $G / N$ is finite, though $G /(N, G)$ is infinite, showing the indispensability of the second condition.

If $G$ is infinite and $N=1$, then ${ }_{i-1} N /{ }_{i} N$ is finite for every $i$, showing that the finiteness of $G / N$ is not a necessary condition for the finiteness of the groups ${ }_{i-1} N /{ }_{i} N$.

Remark 4. In $\$ 6$ we shall obtain a more general theorem of which Corollary 4 will be shown to be a special case.

By letting $N=(G, G)$ in Corollary 4 we obtain the following interesting special case.

Corollary $4^{\prime}$. All the quotient groups $G /{ }^{i} G$ are finite if, and only if, $G /(G, G)$ is finite.

As an application of Corollary $4^{\prime}$ we prove a characterization of finite nilpotent groups. Let us note first the well known facts that a finite group $G$ is termed nilpotent whenever $G$ is the direct product of $p$-groups, and that a finite group $G$ is nilpotent if, and only if, $G$ is of finite class $\left({ }^{i} G=1\right.$ for some $i$ ).

THEOREM 4. The group $G$ is a finite nilpotent group if, and only if, $G /(G, G)$ is finite and there exists an integer $i$ such that ${ }^{i} G=1$.

Proof. The necessity of our conditions is an obvious consequence of the classical results mentioned just now. If conversely the conditions are satisfied, then it follows from Corollary $4^{\prime}$ that $G=G /{ }^{i} G$ is a finite group, and that finite groups of finite class are nilpotent, as has been mentioned before.

4. The generating bilinear form of ${ }_{i} N /{ }_{i+1} N$. If $u$ is an element in $G$ and $v$ 
an element in ${ }_{i-1} N$, for positive $i$, then their commutator $(u, v)$ belongs to ${ }_{i} N$, and ${ }_{i} N$ is generated by these commutators. Since ${ }_{i} N /{ }_{i+1} N$ is part of the center of $G /{ }_{i+1} N$, it is possible to verify the following congruences $\left({ }^{14}\right)$ :

$$
\begin{aligned}
& \left(u, v^{\prime} v^{\prime \prime}\right) \equiv\left(u, v^{\prime}\right)\left(u, v^{\prime \prime}\right) \text { modulo }{ }_{i+1} N, \text { for } v^{\prime}, v^{\prime \prime} \text { in }{ }_{i-1} N ; \\
& \left(u^{\prime} u^{\prime \prime}, v\right) \equiv\left(u^{\prime}, v\right)\left(u^{\prime \prime}, v\right) \text { modulo }{ }_{i+1} N, \text { for } v \text { in }{ }_{i-1} N .
\end{aligned}
$$

From formula (3) and from the definition of ${ }_{i+1} N$ we deduce

$$
\left((G, G),{ }_{i-1} N\right)=\left({ }^{1} G,{ }_{i-1} N\right) \leqq{ }_{i+1} N=\left(G,{ }_{i} N\right) .
$$

Hence it follows from $\left(6^{\prime}\right)$ that

$$
\begin{aligned}
& u^{\prime} \equiv u^{\prime \prime} \text { modulo }(G, G) \text { implies }\left(u^{\prime}, v\right) \equiv\left(u^{\prime \prime}, v\right) \text { modulo }_{i+1} N ; \\
& v^{\prime} \equiv v^{\prime \prime} \text { modulo }{ }_{i} N \text { implies }\left(u, v^{\prime}\right) \equiv\left(u, v^{\prime \prime}\right) \text { modulo }_{i+1} N ;
\end{aligned}
$$

provided $v, v^{\prime}, v^{\prime \prime}$ are in ${ }_{i-1} N$.

We define the generating bilinear form $b_{i}(x, y)$ of ${ }_{i} N /{ }_{i+1} N$ by the formula :

$$
b_{i}\left((G, G) u,{ }_{i} N v\right)={ }_{i+1} N(u, v)
$$

for $u$ in $G$ and $v$ in ${ }_{i-1} N$. It is a consequence of $\left(6^{\prime \prime}\right)$ that $b_{i}(x, y)$ is a singlevalued function of its two variables, the first variable ranging over $G /(G, G)$ and the second one over ${ }_{i-1} N /{ }_{i} N$. It has been remarked before that the values of $b_{i}(x, y)$ generate ${ }_{i} N /{ }_{i+1} N$, and the bilinearity of $b_{i}(x, y)$ is contained in $\left(6^{\prime}\right)$.

The forms $b_{i}$ meet further requirements beyond those already enumerated. We mention two.

$$
b_{1}\left((G, G), w,{ }_{1} N w\right)=1 \quad \text { for } w \text { in } N .
$$

This is an obvious consequence of $(w, w)=1$. It clearly cannot be deduced from the bilinearity, since $N$ may contain elements $w$ which are not contained in $(G, G)$.

$$
b_{i}(x, y)=1 \text { for } x \text { in } N(G, G) /(G, G) \text { and } y \text { in }\left[{ }_{i-1} N \cap{ }^{i} G\right] /{ }_{i} N .
$$

We deduce from formula (3) the inequality $\left(N,{ }^{i} G\right) \leqq{ }_{i+1} N$, and $\left(7^{\prime \prime}\right)$ is an immediate consequence of this inequality. Again it is clear that $\left(7^{\prime \prime}\right)$ cannot be derived from the bilinearity of $b_{i}$.

From the definitions of ${ }_{i} N$ and ${ }^{i} G$ one readily deduces the following inequality.

$$
b_{i}\left(G /(G, G),\left({ }_{i-1} N \cap{ }^{i} G\right) /{ }_{i} N\right) \leqq\left({ }_{i} N \cap{ }^{i+1} G\right) /{ }_{i+1} N \text { for } 0<i .
$$

It would be interesting to obtain general criteria for equality.

The following theorem takes up a question touched upon at the end of $\$ 2$ which will be of importance for an application in $\$ 7$.

Theorem. Suppose that $N$ is a normal subgroup of the free group $G$ and

(14) Baer [4], Hall [1]. 
that ${ }^{(15)} 0<i$. Put $E=G /{ }_{i} N$ and denote by $E^{*}$ the group which is generated by the free central factor set $f(x, y)$ of $E$ and by $F$ the free central extension of $E^{*}$ by E. Then:

(a) $F$ is the direct product of a subgroup $S$ of $E^{*}$ and of a group $T$ satisfying $T \cap E^{*}=E^{*} \cap(F, F)$.

(b) If the subgroups $S$ and $T$ of $F$ meet the requirement (a), then there exists an isomorphism of $T$ upon $G /{ }_{i+1} N$ which induces the identity in $E=T /\left(T \cap E^{*}\right)$ and which maps $T \cap E^{*}$ upon ${ }_{i} N /{ }_{i+1} N$.

(c) If $t(x, y)$ is a central factor set of $E$ in ${ }_{i} N /{ }_{i+1} N$ which defines the central extension $G /{ }_{i+1} N$ of ${ }_{i} N /{ }_{i+1} N$ by $E$, and if $s(x, y)$ is a central factor set of $E$ in the abelian group $Z$, then there exists a homomorphism $\eta$ of ${ }_{i} N /{ }_{i+1} N$ into $Z$ such that $s(x, y)$ and $t(x, y)^{n}$ are associate in $Z$.

Proof. From $0<i$ we infer ${ }_{i} N \leqq(G, G)$. Hence $(E, E)=(G, G) /{ }_{i} N$ so that $E /(E, E)$ and $G /(G, G)$ are isomorphic groups. But the latter one is a free abelian group, since $G$ is a free group. Hence $E /(E, E)$ is a free abelian group.

From $0<i$ we infer that $M={ }_{i} N /{ }_{i+1} N$ is generated by the values of the generating bilinear form $b_{i}(x, y)$ for $x$ in $G /(G, G)$ and $y$ in ${ }_{i-1} N /{ }_{i} N$. Consequently $H=G /{ }_{i+1} N$ is generated by a set of representatives of the cosets, not $M$, of $H / M$. Hence we infer from Theorem 1 of $\$ 1$ the existence of a central factor set $h(x, y)$ of $E$ in $M$ which defines the central extension $H$ of $M$ by $E$ and whose values generate $M$.

Since $F / E^{*}(F, F)$ and $E /(E, E)$ are essentially the same groups, we infer from the result of the first paragraph of this proof that $F / E^{*}(F, F)$ is a free abelian group. Since $F$ is the free central extension by $E$, it follows from a remark made at the beginning of $\S 3$ that $F /(F, F)$ is a free abelian group. Hence we may infer from Corollary 2 of $\$ 2$ the existence of subgroups $S$ and $T$ of $F$ which meet the requirements of (a). Thus (a) is proven. Let us note the following consequence of Theorem 3 of $\S 2:$ If $E^{*}$ is the direct product of $E^{*} \cap(F, F)$ and a subgroup $S$, then there exists a subgroup $T$ such that $S$ and $T$ meet the requirements of (a).

Assume now that $S$ and $T$ are any couple of subgroups of $F$ which meet the requirements of (a). Since $E^{*}$ is commutative, so is $S$. Hence $(F, F) \leqq T$. Since $f(x, y)$ is the free central factor set of $E$, and since $M$ is generated by the values of the central factor set $h(x, y)$ of $E$ in $M$, there exists a homomorphism of $E^{*}$ upon $M$ which maps the element $f(x, y)$ upon $h(x, y)$. It is readily verified (see the proof of Lemma 2 of $\$ 2$ ) that there exists a homomorphism $\alpha$ of $F$ upon $H$ which induces this homomorphism of $E^{*}$ upon $M$ and which induces the identity in $E$. Thus $F^{\alpha}=H, E^{* \alpha}=M$ and $f(x, y)^{\alpha}$ $=h(x, y)$. Since $G$ is a free group, it is easy to construct a homomorphism $\phi$ of $G$ into $F$ which induces the identity in $E$. Clearly ${ }_{i} N^{\phi} \leqq E^{*}$. But $E^{*}$ is part

(15) The situation is altogether different for $i=0$. A discussion of this case is given in Corollary 2 of $\$ 2$. 
of the center of $F$ and hence ${ }_{i+1} N^{\phi}=1$. Thus a homomorphism $\beta$ of $H$ into $F$ is induced by $\phi$, and $\beta$ induces the identity in $E$ and maps $M$ into $E^{*}$. This implies in particular $F=E^{*} H^{\beta}$ and $M^{\beta}=E^{*} \cap H^{\beta}$. If an element belongs to a coset of $H / M$ which corresponds to the element $x$ of $E$, then it is mapped by $\beta$ upon an element in $F$ whose coset modulo $E^{*}$ corresponds to $x$ too, and a like statement applies to $\alpha$. Thus reciprocal isomorphisms between $H / M$ and $F / E^{*}$ are induced by $\beta$ and $\alpha$. Since $(H, M)=1$ and $\left(F, E^{*}\right)=1$, this implies $\left({ }^{16}\right)$ that $\beta$ and $\alpha$ induce reciprocal isomorphisms between $(H, H)$ and $(F, F)$. From $0<i$ we infer ${ }_{i} N \leqq(G, G)$ and therefore $M \leqq(H, H)$. From $(F, F) \leqq T$ we deduce now $M \leqq(H, H)=(F, F)^{\alpha} \leqq T^{\alpha}$. Since elements in $T$ represent all the cosets of $F / E^{*}$, and since $\alpha$ induces an isomorphism of $F / E^{*}$ upon the whole group $H / M$, every coset of $H / M$ is represented by elements in $T^{\alpha}$, and thus we have shown that $H=T^{\alpha}$. If the element $w$ in $T$ is mapped upon 1 by $\alpha$, then the coset $E^{*} w$ is mapped upon 1 by the isomorphism upon $H / M$ which $\alpha$ induces in $F / E^{*}$. Hence $E^{*}=E^{*} w$ and $w$ belongs to $E^{*}$. But $T \cap E^{*}=E^{*} \cap(F, F)$, and $\alpha$ has been shown to induce an isomorphism in $(F, F)$. Hence $w^{\alpha}=1$ for $w$ in $T$ implies $w=1$, proving that $\alpha$ is an isomorphism of $T$ upon $H$ which meets all the requirements of (b), since $E^{*}$ is mapped by $\alpha$ upon $M$. This completes the proof of (b).

If $t(x, y)$ is a central factor set of $E$ in $M$ which defines the central extension $H$ of $M$ by $E$, then $l(x, y)^{\alpha^{-1}}$ is a central factor set of $E$ in $(F, F) \cap E^{*}$ $=E^{*} \cap T$ which defines the central extension $T$ of $E^{*} \cap T$ by $E$. Since $F$ is the direct product of $T$ and of a subgroup $S$ of $E^{*}$, it follows now that $f(x, y)$ and $t(x, y)^{\alpha^{-1}}=r(x, y)$ are associate central factor sets of $E$ in $E^{*}$. If $Z$ is some abelian group, and if $s(x, y)$ is a central factor set of $E$ in $Z$, then there exists a homomorphism $\kappa$ of $E^{*}$ into $Z$ such that $f(x, y)^{*}=s(x, y)$ for $x, y$ in $E$, since $f(x, y)$ is the free central factor set of $E$. But this implies that $r(x, y)^{\kappa}$ and $s(x, y)$ are associate central factor sets of $E$ in $Z$. Thus $\alpha^{-1} \kappa$ induces a homomorphism of $M$ into $Z$ which maps $t(x, y)$ upon the factor set $r(x, y)^{x}$ which is associate to $s(x, y)$ in $Z$, and this completes the proof of (c) and of the theorem.

5. Properties transmitted through the generating bilinear forms. The abelian group $A$ is said( $\left({ }^{17}\right)$ to be of rank not greater than $n$ if every subgroup of $A$ which can be generated by a finite number of elements may be generated by $n$ of its elements.

Theorem 1. (a) If both $G /(G, G)$ and $N /(N, G)$, for $N$ a normal subgroup of $G$, are generated by a finite number of elements, then every ${ }_{i} N /{ }_{i+1} N$ is generated by a finite number of elements.

(b) If the rank of $G /(G, G)$ is not greater than $m$, and if the rank of $N /(N, G)$ is not greater than $n$, then the rank of ${ }_{i} N /{ }_{i+1} N$ does not exceed $m^{i} n$.

(16) See Baer $[4, \$ 4]$ for a sketch of a proof of this fact.

(17) This concept is due to Prüfer. 
Proof. If $S$ is a set of elements generating $G /(G, G)$ and if ${ }_{i-1} N /{ }_{i} N$ is generated by its subset $T$, then ${ }_{i} N /{ }_{i+1} N$ is generated by the set of elements $b_{i}(s, t)$ for $s$ in $S$ and $t$ in $T$, since $b_{i}(x, y)$ is, by the results of the preceding section, a bilinear form whose values generate ${ }_{i} N /{ }_{i+1} N$. If both sets $S$ and $T$ are finite, then ${ }_{i} N /{ }_{i+1} N$ is therefore generated by a finite number of elements; now it is clear how to prove the statement (a) by complete induction with regard to $i$.

The statement (b) is certainly true for $i=0$, and thus we may make the induction hypothesis that the rank of ${ }_{i-1} N /{ }_{i} N$, for $0<i$, does not exceed $m^{2-1} n$. If $S$ is a finite subset of ${ }_{i} N /{ }_{i+1} N$, then there exists a finite subset $S^{\prime}$ of $G /(G, G)$ and a finite subset $S^{\prime \prime}$ of ${ }_{i-1} N /{ }_{i} N$ such that every element in $S$ is a product of elements $b_{i}\left(s^{\prime}, s^{\prime \prime}\right)$ for $s^{\prime}$ in $S^{\prime}$ and $s^{\prime \prime}$ in $S^{\prime \prime}$. There exists a subset $T^{\prime}$ of $G /(G, G)$ which contains not more than $m$ elements such that every element in $S^{\prime}$ is a product of elements in $T^{\prime}$, and likewise there exists a subset $T^{\prime \prime}$ of ${ }_{i-1} N /{ }_{i} N$ which contains not more than $m^{i-1} n$ elements and such that every element in $S^{\prime \prime}$ is a product of elements in $T^{\prime \prime}$. Clearly $S$ is contained in the subgroup generated by all the elements $b_{i}\left(t^{\prime}, t^{\prime \prime}\right)$ for $t^{\prime}$ in $T^{\prime}$ and $t^{\prime \prime}$ in $T^{\prime \prime}$, and this subgroup is generated by not more than $m^{i} n$ elements. The rank of ${ }_{i} N /{ }_{i+1} N$ is consequently not greater than $m^{i} n$, as we intended to show.

Remark 1. Since the subgroup $(G, G) N /(G, G)$ of $G /(G, G)$ and the quotient group $N /[N \cap(G, G)]$ of $N /(N, G)$ are isomorphic groups, it suffices to assume in Theorem 1 that the three groups $G /(G, G) N,(G, G) N /(G, G)$ and $[N \cap(G, G)] /(N, G)$ are generated by a finite number of elements or are of finite rank.

Remark 2. The impossibility of omitting the hypothesis on $N /(N, G)$ in Theorem 1 may be seen from the following example.

Denote by $B$ a direct product of a countable infinity of infinite cyclic groups, and denote by $b(1), b(2), \cdots, b(i), \cdots$ a basis of $B$. Let $C$ be the group obtained by adjoining to $B$ elements $z(i)$, for $i=0, \pm 1, \pm 2, \cdots$, subject to the following relations:

$$
z(i) x=x z(i) \text { for } x \text { in } B, \quad(z(i), z(i+j))=b(j) \text { for } 0<j .
$$

Then $(z(i), z(i-j))=(z(i-j), z(i))^{-1}=b(j)^{-1}$ for $0<j$. It is readily verified $\left(^{18}\right)$ that $B$ is part of the center of $C$, and that the elements $z(i)$ represent a basis of the free abelian group $C / B$.

Since $(z(i), z(j))=(z(i+1), z(j+1))$ for every pair $i, j$, there exists one and only one automorphism $\alpha$ of $C$ such that

$$
x^{\alpha}=x \text { for } x \text { in } B \text { and } z(i)^{\alpha}=z(i+1) \text { for every } i .
$$

We denote by $G$ the group obtained by adjoining to $C$ an element $z$, subject to the relations

(18) Baer [3]. 


$$
z^{-1} y z=y^{\alpha} \text { for } y \text { in } C .
$$

It is clear that $C$ is a normal subgroup of $G$, that $G / C$ is an infinite cyclic group, and that $B$ is part of the center of $G$. Thus $B /(G, B)=B$ is a direct product of an infinity of infinite cyclic groups and is certainly not of finite rank.

The group $G$ is generated by the elements $z, z(i), b(j)$. But $z(i)=z^{-i} z(0) z^{i}$ and $b(j)=(z(0), z(j))$ for $0<j$. Hence $G$ is generated by the two elements $z$ and $z(0)$, and clearly $G /(G, G)$ is generated by two elements. Thus we have constructed a group $G$ and a normal subgroup $B$ of $G$ such that $G$ and $G /(G, G)$ are generated by two elements, though $B /(G, B)$ is not of finite rank.

Remark 3. The impossibility of omitting the hypothesis on $G /(G, G)$ in Theorem 1 may be seen from the following example.

Denote by $B$ a direct product of a countable infinity of infinite cyclic groups, and denote by $b(1), b(2), \cdots, b(i), \cdots$ a basis of $B$. Let $G$ be the group obtained by adjoining to $B$ elements $a(0), a(1), \cdots, a(i), \cdots$ subject to the following relations :

$$
\begin{aligned}
a(i) x & =x a(i) \text { for } x \text { in } B \text { and } 0 \leqq i ; \\
a(i) a(j) & =a(j) a(i) \text { for } 0<i \leqq j ; \\
(a(0), a(j)) & =b(j) \text { for } 0<j .
\end{aligned}
$$

It is readily verified $\left({ }^{18}\right)$ that $B$ is at the same time the center and the commutator subgroup of $G$, and that $G / B$ is a free abelian group a basis of which is represented by the elements $a(i)$.

Denote by $N$ the subgroup of $G$ which is generated by $a(0)$ and $B$. Clearly $N$ is a normal subgroup of $G$ and $(G, N)=B$. Thus $N /(G, N)$ is an infinite cyclic group. But ${ }_{2} N=(G, B)=1$, and thus ${ }_{1} N /{ }_{2} N$ is of infinite rank. Consequently we have constructed a normal subgroup $N$ of a group $G$ such that $N /(G, N)$ is a cyclic group, though ${ }_{1} N /{ }_{2} N$ is a free abelian group of infinite rank.

Remark 4. If $N$ is a normal subgroup of the free group $G$, then every free set of generators of $G$ leads to a representation of $G / N$ by means of generators and relations, and a "complete" set of relations between these generators may be obtained by considering a set of generators of $N /(G, N)$. Thus (a) of Theorem 1 may be applied, whenever $G / N$ is obtained from a representation of the group $G / N$ by means of a finite number of generators and a finite number of relations.

Corollary 1. (a) If $G /(G, G)$ is generated by a finite number of elements, then ${ }^{i} G /{ }^{i+1} G$, for $0 \leqq i$, is generated by a finite number of elements.

(b) If the rank of $G /(G, G)$ is not greater than $m$, then the rank of ${ }^{i} G /{ }^{i+1} G$, for $0 \leqq i$, does not exceed $m^{i+1}$.

This is an immediate consequence of Theorem 1, and is obtained by selecting in particular $N=G$. 
It is of interest to note that in (a) we need not make the hypothesis that $G$ itself be generated by a finite number of elements. Thus (a) improves upon a well known result.

A group has been said to be a $Q$-group if it is not isomorphic to a proper quotient group. In particular abelian groups are $Q$-groups whenever they are generated by a finite number of elements. Applying a simple criterion $\left({ }^{19}\right)$, we deduce now from (a) of Corollary 1 the following fact.

Corollary 2. If 1 is the cross cut of the subgroups ${ }^{i} G$, and if $G /(G, G)$ is generated by a finite number of elements, then $G$ is a $Q$-group.

If we say that an element is of order 0 if it generates an infinite cyclic group, then one verifies immediately that the order of $b_{i}(x, y)$ is a common divisor of the orders of $x$ and $y$. For $b_{i}$ is bilinear. An immediate consequence of this fact is the following statement.

THEOREM 2. If ${ }_{i} N /{ }_{i+1} N$, for $0<i$, contains elements of order 0 , then both $G /(G, G)$ and ${ }_{i-1} N /{ }_{i} N$ contain elements of order 0. If ${ }_{i} N /{ }_{i+1} N$ contains elements of order a prime number $p$, then the absence of elements of order 0 implies the presence of elements of order $p$, both in $G /(G, G)$ and in ${ }_{i-1} N /{ }_{i} N$.

An abelian group is of finite order if it is generated by a finite number of elements whose orders are different from 0 . Hence we may deduce from (a) of Theorem 1 and from Theorem 2 the following supplement to Theorem 3 of $\S 3$.

Corollary 3. If both $G /(G, G)$ and $N /(N, G)$ are finite groups, then every ${ }_{i} N /{ }_{i+1} N$, for $0 \leqq i$, is finite.

In the special case $N=G$ we prove a slightly more precise result. A group is termed a $p$-group if all its elements are of order a power of $p$.

CoRollary 4. If all the elements in $G /(G, G)$ are of finite order, then $G /{ }^{i} G$, for positive $i$, is a direct product of p-groups.

Proof. It is an immediate consequence of Theorem 2 that the orders of the elements in ${ }^{i-1} G /{ }^{i} G$ are finite. This implies clearly, by complete induction with regard to $i$, that the orders of the elements in $G /{ }^{i} G$ are finite. Now it follows from a theorem on nilpotent groups $\left({ }^{20}\right)$ that $G /{ }^{i} G$ is a direct product of $p$-groups. Note that the same prime numbers are orders of elements in $G /{ }^{i} G$ that are orders of elements in $G /(G, G)$.

6. The finiteness of the groups ${ }^{i} G /{ }_{i} N$. In order to obtain the main results of this section we need certain generalizations of previous concepts and results which may be of some interest independent of their application.

(19) Baer [5, Theorem 2].

(20) Baer [4, Appendix II]. 
The group $G$ is said to be a centralizer extension of the group $N$ by the group $E$, if :

(i) $N$ is a normal subgroup of the group $G$.

(ii) $G / N$ and $E$ are isomorphic groups.

(iii) Every coset of $G / N$ contains at least one element that permutes with every element in $N$.

If we denote by $Z(N<G)$ the centralizer of $N$ in $G$, that is, the set of all the elements in $G$ which permute with every element in $N$, then condition (iii) may be written shortly as

$$
G=N Z(N<G)
$$

We note furthermore that condition (i) is a consequence of (iii).

If $G$ is a centralizer extension of the group $N$ by the group $E$, then we may select in the coset of $G / N$ which corresponds to the element $x$ in $E$ a representative $r(x)$ which permutes with every element in $N ; r(1)=1$. Denote by $R$ the subgroup of $G$ which is generated by the center of $N$ and by the elements $r(x)$. It is readily verified that $R$ is a central extension of the center $Z$ of $N$ by $E$, and that the elements $f(x, y)=r(x) r(y) r(x y)^{-1}$ form a central factor set of $E$ in $Z$ which defines $R$ and $G$. Thus every centralizer extension of $N$ by $E$ is defined by a central factor set of $E$ in the center of $N$.

Any other set of representatives of the cosets of $G / N$ may be written in the form $t(x) r(x)$ for $t(x)$ in $N$ and $t(1)=1$. Since the elements $r(x)$ permute with every element in $N$, we obtain $[t(x) r(x)][t(y) r(y)][t(x y) r(x y)]^{-1}$ $=t(x) t(y) t(x y)^{-1} f(x, y)$. Consequently we term every function $t(x, y)$ $=t(x) t(y) t(x y)^{-1}$ with $t(x)$ in $N$ and $t(1)=1$ a centralizer transformation set of $E$ in $N$. An extension $G$ of a group $N$ by a group $E$ is called $a$ splitting extension if there exists a subgroup $\left.{ }^{21}\right) V$ of $G$ such that $G=N V$ and $N \cap V=1$. It is clear that the centralizer extension $G$ of the group $N$ by the group $E$ splits if, and only if, the central factor set of $E$ in the center of $N$ which defines this extension is a centralizer transformation set of $E$ in $N$.

THEOREM 1. If $G$ is a centralizer extension of the group $N$ by the group $E$, then ${ }^{i} N={ }_{i} N$ for $0 \leqq i$, and if $G=N S$ is satisfied by the subgroup $S$ of $G$, then ${ }^{i} G={ }^{i} N i S$ for $0 \leqq i$.

Proof. Since ${ }^{0} N={ }_{0} N=N$, we may assume the validity of ${ }^{i-1} N={ }_{i-1} N$ in order to prove the equation ${ }^{i} N={ }_{i} N$. From this induction hypothesis we deduce ${ }^{i} N=\left(N,{ }^{i-1} N\right)=\left(N,{ }_{i-1} N\right) \leqq\left(G,{ }_{i-1} N\right)={ }_{i} N$. If furthermore $u$ is an element in $G$ and $v$ an element in ${ }_{i-1} N$, then there exist elements $u^{\prime}, u^{\prime \prime}$ in $N$ and in the centralizer of $N$ respectively, such that $u=u^{\prime} u^{\prime \prime}$. Consequently $(u, v)=\left(u^{\prime}, v\right)$, since $v$ belongs to ${ }_{i-1} N \leqq N$. Hence ${ }_{i} N=\left(G,{ }_{i-1} N\right) \leqq\left(N,{ }_{i-1} N\right)$ $=\left(N,{ }^{i-1} N\right)={ }^{i} N$, proving our first contention ${ }_{i} N={ }^{i} N$.

Since ${ }^{0} N^{0} S=N S=G={ }^{0} G$, we may assume the validity of ${ }^{i-1} G={ }^{i-1} N^{i-1} S$

(21) It is important to notice that $V$ need not be a normal subgroup of $G$. 
in order to prove the equation ${ }^{i} G={ }^{i} N^{i} S$. Every element $x$ in $G$ has the form $x^{\prime} x^{\prime \prime}$ for $x^{\prime}$ in $N$ and $x^{\prime \prime}$ in $S$, and every element $y$ in ${ }^{i-1} G$ has, because of the induction hypothesis, the form $y^{\prime} y^{\prime \prime}$ for $y^{\prime}$ in ${ }^{i-1} N$ and $y^{\prime \prime}$ in ${ }^{i-1} S$. One readily verifies the validity of the following identity:

$$
(x, y)=x^{\prime \prime-1}\left(x^{\prime}, y^{\prime \prime}\right) x^{\prime \prime}\left(x^{\prime \prime}, y^{\prime \prime}\right)\left(x^{\prime \prime} y^{\prime \prime}\right)^{-1}\left(x^{\prime}, y^{\prime}\right)\left(x^{\prime \prime} y^{\prime \prime}\right) y^{\prime \prime-1}\left(x^{\prime \prime}, y^{\prime}\right) y^{\prime \prime} \text {. }
$$

It is clear that $\left(x^{\prime \prime}, y^{\prime \prime}\right)$ belongs to $\left(S,{ }^{i-1} S\right)=i S$, and that both $\left(x^{\prime}, y^{\prime}\right)$ and $\left(x^{\prime \prime} y^{\prime \prime}\right)^{-1}\left(x^{\prime}, y^{\prime}\right)\left(x^{\prime \prime} y^{\prime \prime}\right)$ belong to the normal subgroup $\left(N,{ }^{i-1} N\right)={ }^{i} N$. From formula (3) and the equation ${ }^{i} N={ }_{i} N$ we deduce that both $\left(x^{\prime}, y^{\prime \prime}\right)$ and $x^{\prime \prime-1}\left(x^{\prime}, y^{\prime \prime}\right) x^{\prime \prime}$ belong to $\left(N,{ }^{i-1} S\right) \leqq\left(N,{ }^{i-1} G\right) \leqq{ }_{i} N={ }^{i} N$, and likewise we see that both $\left(x^{\prime \prime}, y^{\prime}\right)$ and $y^{\prime \prime-1}\left(x^{\prime \prime}, y^{\prime}\right) y^{\prime \prime}$ belong to $\left(S,{ }^{i-1} N\right) \leqq\left(G,{ }_{i-1} N\right)={ }_{i} N$ $={ }^{i} N$. Thus we have shown that $(x, y)$ belongs to ${ }^{i} S^{i} N$ if $x$ is in $G$ and $y$ in ${ }^{i-1} G$. Hence ${ }^{i} G=\left(G,{ }^{i-1} G\right) \leqq{ }^{i} S^{i} N \leqq{ }^{i} G$, proving the desired equality.

Following P. Hall( $\left.{ }^{22}\right)$ we term a group $G$ of class $n$, if ${ }^{n} G=1$.

COROLlaRY 1. If $G$ is a splitting centralizer extension of the group $N$, and if $N$ is of class $i$, then ${ }^{i} G \cap N=1$.

Proof. There exists a subgroup $S$ of $G$ such that $G=S N$ and $1=S \cap N$. Furthermore ${ }^{i} N=1$. Hence it follows from Theorem 1 that ${ }^{i} G \cap N={ }^{i} S^{i} N \cap N$ $=i S \cap N \leqq S \cap N=1$, proving our contention.

Suppose now that $G$ is a central extension of the group $M$ by the group $E$, determined by the central factor set $f(x, y)$ of $E$ in $M$. If $M$ is furthermore part of the center of the group $N$, then there exists one and essentially only one centralizer extension $H$ of $N$ by $E$ which is determined by $f(x, y)$. We may assume without loss in generality that $H=G N, M=G \cap N$ and that every element in $G$ permutes with every element in $N$. If in particular $H$ is a splitting extension of $N$, then we say that $N$ splits the central extension $G$ of $M$ by $E$. Using a result stated at the beginning of this section we see that $N$ splits the central extension $G$ of $M$ by $E$ if, and only if, $f(x, y)$ is a centralizer transformation set of $E$ in $N$. This implies in particular that every central extension is split by itself. If there exists a group $M$ of class $i$ which splits the central extension $G$ of $M$ by $E$, then we say that this central extension is $i$-splitting.

CoROLlaRY $1^{\prime}$. If $G$ is an $i$-splitting central extension of $M$, then ${ }^{i} G \cap M=1$.

Proof. There exists a group $S$ of class $i$ which splits the central extension $G$ of $M$. If $H$ is the centralizer extension of $S$ such that $H=G S, M=G \cap S$ and such that every element in $G$ permutes with every element in $S$, then $H$ is a splitting centralizer extension of $S$, and it follows from Corollary 1 that ${ }^{i} G \cap M \leqq{ }^{i} H \cap S=1$, proving our contention.

Extending Corollary 1 of $\$ 2$ we prove now the following theorem.

(22) According to the terminology introduced by P. Hall [1, p. 50] a group $G$ would be termed of class not greater than $n$ if it satisfies ${ }^{n} G=1$. But for our purposes the present slightly changed terminology seems to be more convenient. 
ThEOREM 2. If the central extension $G$ of the group $Z$ by the group $E$ is determined by the central factor set $f(x, y)$ of $E$ in $Z$, then the following properties of the homomorphism $\eta$ of $Z$ imply each other.

(i) $\left({ }^{i} G \cap Z\right)^{\eta}=1$.

(ii) $f(x, y)^{\eta}$ determines an i-splitting central extension of $Z^{\eta}$ by. $E$.

Proof. Denote by $H$ a central extension of $Z^{\eta}$ by $E$ which realizes the central factor set $f(x, y)^{\eta}$ of $E$ in $Z \eta$, and denote by $\phi$ a homomorphism of $G$ upon $H$ which induces $\eta$ in $Z$ and the identity in $E$. The existence of $H$ and $\phi$ are readily deduced from Lemma 2 of $\$ 2$. Clearly ${ }^{i} G$ is mapped by $\phi$ upon ${ }^{i} H$ and the kernel of $\phi$ is part of $Z$. Consequently

$$
\left({ }^{i} G \cap Z\right)^{\eta}={ }^{i} H \cap Z Z^{\eta} \text {. }
$$

Suppose now that (i) is satisfied by $\eta$. Denote by $\gamma$ the homomorphism of $G$ upon $H /{ }^{i} H$ which maps the element $v$ in $G$ upon the element ${ }^{i} H v^{\phi}$ in $H /{ }^{i} H$. Since ${ }^{i} H \cap Z^{\eta}=1$, it follows that $\gamma$ induces $\eta$ in $Z$. Thus $H /{ }^{i} H$ is a central extension of $Z^{\eta}$, is of class $i$, and $f(x, y) \eta$ is clearly a centralizer transformation set of $E$ in $H /{ }^{i} H$. But this is equivalent to the property (ii) which is consequently implied by (i).

Suppose conversely that (ii) is satisfied by $\eta$. Then there exists a group $S$ of class $i$ such that $Z^{\eta}$ is part of the center of $S$ and such that $S$ splits $H$. Consider the centralizer extension $H S$ of $S$ which satisfies $H \cap S=Z$ n. Since this is a splitting extension, we may deduce from Corollary 1 the equation ${ }^{i}(H S) \cap S$ $=1$. This implies in particular that

$$
\left({ }^{i} G \cap Z\right)^{\eta}={ }^{i} H \cap Z^{\eta} \leqq i(H S) \cap S=1,
$$

showing that (i) is a consequence of (ii), as we intended to prove.

If $N$ is a normal subgroup of the group $G$, then $G /{ }_{i+1} N$ is a central extension of ${ }_{i} N /{ }_{i+1} N$ by $G /{ }_{i} N$, and Theorem 2 may serve to characterize the subgroup $\left[{ }^{i+1} G \cap{ }_{i} N\right] /{ }_{i+1} N$. To make this useful in our applications we shall have to derive from this central extension a central extension of ${ }_{i} N /{ }_{i+1} N$ by $G /{ }^{i} G$, and to this problem our next considerations are devoted.

TheOREM 3. If $f(x, y)$ is a central factor set of the group $E$ in the abelian group $Z$, if the normal subgroup $F$ of $E$ is of finite order $n$, then there exists a central extension $C$ of $Z$ by $E / F$ such that $f(x, y)^{n}$ is a centralizer transformation set of $E$ in $C$.

Proof $\left({ }^{23}\right)$. Since $F$ is finite, we may put for any pair $x, y$ of elements in $E$

$$
d(x, y)=\prod_{z \in F} f(z x, y) f(z, y)^{-1} .
$$

Since $F$ is a normal subgroup of $E$, the elements $z x$ range over a full coset $F x=x F$ of $E / F$. Hence $d(x, y)=d(F x, y)$. Since $Z$ is commutative, and since

(23) The main construction of this proof has been used before for similar purposes. 
$f(x z, y) f(z, y)^{-1}=f(x, z y) f(x, z)^{-1}$ is satisfied by every central factor set of $E$ in $Z$, we find

$$
d(x, y)=\prod_{z \in F} f(x, z y) f(x, z)^{-1},
$$

proving that $d(x, y)=d(x, F y)$. Thus a single-valued function $f_{F}(X, Y)$ of the elements $X$ and $Y$ in $E / F$ is defined by $f_{F}(F x, F y)=d(x, y)$. It is clear that $d(1, y)=d(x, 1)=1$, and that therefore $f_{F}(1, Y)=f_{F}(X, 1)=1$. If the elements $x, y, z$ in $E$ belong to the cosets $X, Y, Z$ respectively, then $x y$ belongs to $X Y$ and so on, and we find:

$$
\begin{aligned}
f_{F}(X, Y) f_{F}(X Y, Z) & =\prod_{t \in F} f(t x, y) f(t, y)^{-1} f(t x y, z) f(t, z)^{-1} \\
& =\prod_{t} f(y, z) f(t x, y z) f(t, y)^{-1} f(t, z)^{-1} \\
& =\prod_{t} f(t y, z) f(t, y z)^{-1} f(t x, y z) f(t, z)^{-1} \\
& =f_{F}(Y, Z) f_{F}(X, Y Z),
\end{aligned}
$$

proving that $f_{F}(X, Y)$ is a central factor set of $E / F$ in $Z$.

Consequently there exists a central extension $C$ of $Z$ by $E / F$ which is determined by this central factor set. It is therefore possible to select in the coset of $C / Z$ which corresponds to the element $X$ in $E / F$ a representative $r(X)$ such that $r(1)=1$ and $f_{F}(X, Y)=r(X) r(Y) r(X Y)^{-1}$.

Put $t(x)=r(F x) \prod_{2 \in F} f(x, z)$. This is a single-valued function of the elements in $E$, since $F$ is finite, and clearly $t(1)=1$. Furthermore we note again that $x z$ and $z x$ both range over the coset $x F=F x$ if $z$ ranges over $F$, and hence it follows from the fundamental identity (5) of central factor sets that

$$
\begin{aligned}
t(u) t(v) t(u v)^{-1} & =r(F u) r(F v) r(F u v)^{-1} \prod_{z \in P} f(u, z) f(v, z) f(u v, z)^{-1} \\
& =f_{F}(F u, F v) \prod_{z} f(u, z) f(v, z) f(u v, z)^{-1} \\
& =\prod_{z} f(z u, v) f(z, v)^{-1} f(u, z) f(v, z) f(u v, z)^{-1} \\
& =\prod_{z} f(u, z) f(u z, v) f(z, v)^{-1} f(v, z) f(u v, z)^{-1} \\
& =\prod_{z} f(u, z v) f(v, z) f(u v, z)^{-1}=\prod_{z} f(u, v)=f(u, v)^{n},
\end{aligned}
$$

and this completes the proof of this theorem.

COROLlary 2. If $G$ is a central extension of the abelian group $Z$ by the group $E$, and if ${ }^{i} E$ is of finite order $n$, then $\left[{ }^{i+1} G \cap Z\right]^{n}=1$.

Proof. Denote by $f(x, y)$ the central factor set of $E$ in $Z$ which determines the central extension $G$ of $Z$ by $E$. Then we deduce from Theorem 3 the exist- 
ence of a central extension $C$ of $Z$ by $E /{ }^{i} E$ such that $f(x, y)^{n}$ is a centralized transformation set of $E$ in $C$. Since $E /{ }^{i} E$ is of class $i$, the group $C$ is of class $i+1$. Mapping every element in $Z$ upon its $n$th power is an endomorphism of $Z$ which maps $f(x, y)$ upon $f(x, y)^{n}$. Since $f(x, y)^{n}$ is a centralized transformation set in a group of class $i+1$, it is a central factor set which determines an $(i+1)$-splitting extension. Hence it follows from Theorem 2 that $\left({ }^{i+1} G \cap Z\right)^{n}=1$, as we desired to prove.

We are now ready to prove the main result of this section.

THEOREM 4. If $N$ is a normal subgroup of the group $G$, and if $G / N$ is a finite group of order $n$, then ${ }^{i} G /{ }_{i} N$ is finite for every $i$, and every prime number dividing the order of ${ }^{i} G /{ }_{i} N$ is a divisor of $n$.

Proof. Denote by $R$ a subgroup of $G$ which is generated by a set of representatives of the cosets, not $N$, of $G / N$, and let $M=R \cap N$. We shall need the following lemma.

(+) $R$ is generated by a finite number of elements, $G / N \sim R / M$, and there exists for every $i \geqq 0$ a homomorphism of ${ }^{i} R /{ }_{i} M$ upon ${ }^{i} G /{ }_{i} N$.

The first assertion of this lemma is obvious, since $R$ is generated by $n-1$ elements. The second assertion follows from $R / M=R /(R \cap N) \sim R N / N$ $=G / N$. To prove the last assertion we note first that ${ }_{i} M \leqq_{i} N$ is an immediate consequence of $M \leqq N$. Since ${ }^{i} R \leqq{ }^{i} G$, it suffices to prove that ${ }^{i} G={ }^{i} R_{i} N$ for every $i$, and this we proceed to do by complete induction with regard to $i$. Since the contention is patently true for $i=0$, we may assume its validity for $i-1$. If $x$ is an element in $G$ and $y$ an element in ${ }^{i-1} G$, then there exists an element $x^{\prime}$ in $R$ and an element $y^{\prime}$ in ${ }^{i-1} R$ such that $x \equiv x^{\prime}$ modulo $N$ and $y \equiv y^{\prime}$ modulo ${ }_{i-1} N$. Clearly $(x, y)$ is an element in ${ }^{i} G$ and $\left(x^{\prime}, y^{\prime}\right)$ is an element in ${ }^{i} R$, and it is readily verified $\left({ }^{24}\right)$ that $(x, y) \equiv\left(x^{\prime}, y^{\prime}\right)$ modulo ${ }_{i} N$. But ${ }^{i} G$ is generated by the commutators $(x, y)$ for $x$ in $G$ and $y$ in ${ }^{i-1} G$, and thus we have shown that ${ }^{i} G={ }^{i} R_{i} N$, completing the proof of the lemma (t).

Since $R / M$ is a finite group, and since $R$ is generated by a set of representatives of the cosets, not $M$, of $R / M$, it follows from Theorem 1 of $\S 1$ and Theorem 12 of $\S 3$ that $M /(R, M)$ is generated by a finite number of elements. Hence we may deduce from Theorem 1 of $\S 5$ that ${ }_{i-1} M /{ }_{i} M$ is, for positive $i$, generated by a finite number of elements.

Clearly ${ }^{0} R /{ }_{0} M=R / M$ is a finite group of order $n(0)=n$, and every prime number dividing $n(0)$ is a divisor of $n$. Hence we may make the induction hypothesis that ${ }^{i-1} R /{ }_{i-1} M$ is a finite group of order $n(i-1)$ and that every prime number dividing $n(i-1)$ is a divisor of $n$. The group $R /{ }_{i} M$ is a central extension of the group $Z={ }_{i-1} M /{ }_{i} M$ by the group $E=R /{ }_{i-1} M$. The group ${ }^{i-1} E$ is essentially the same as the group ${ }^{i-1} R /{ }_{i-1} M$ which is of finite order $n(i-1)$. Consequently we may deduce from Corollary 2 that

(24) By direct computation or by reference to Baer [4] or Hall [1]. 
$\left(Z \cap i\left(R /{ }_{i} M\right)\right)^{n(i-1)}=1$. This implies clearly that the order of every element in $Z \cap i\left(R /{ }_{i} M\right)=\left({ }_{i-1} M \cap{ }^{i} R\right) /{ }_{i} M$ is a divisor of $n(i-1)$. Since ${ }_{i-1} M /{ }_{i} M$ is, as has been shown before, an abelian group generated by a finite number of elements, we have shown that the subgroup $\left({ }_{i-1} M \cap{ }^{i} R\right) / i_{i} M$ of ${ }_{i-1} M /{ }_{i} M$ is an abelian group generated by a finite number of elements whose orders are divisors of $n(i-1)$. Thus $\left({ }_{i-1} M \cap i R\right) /{ }_{i} M$ is certainly a finite group the orders of whose elements are divisors of $n(i-1)$. The group ${ }^{i} R /\left({ }^{i} R \cap_{i-1} M\right)$ is isomorphic to the subgroup $\left({ }_{i-1} M^{i} R\right) /{ }_{i-1} M$ of ${ }^{i-1} R /{ }_{i-1} M$. Since ${ }^{i-1} R /{ }_{i-1} M$ is a finite group of order $n(i-1)$, it follows that ${ }^{i} R /\left({ }^{i} R \cap_{i-1} M\right)$ is a finite group whose order is a divisor of $n(i-1)$. This implies in particular that the order of every element in ${ }^{i} R /\left({ }^{i} R \cap_{i-1} M\right)$ is a divisor of $n(i-1)$. Consequently $i R /{ }_{i} M$ is a finite group the orders of whose elements are divisors of $n(i-1)^{2}$. If $p$ is a prime number dividing the finite order $n(i)$ of ${ }^{i} R /{ }_{i} M$, then it is well known that ${ }^{i} R /{ }_{i} M$ contains an element of order $p$. Hence $p$ is a divisor of $n(i-1)^{2}$ and therefore of $n$. This completes the induction proof. We have actually proved slightly more than we set out to prove, namely the following fact.

(+十) ${ }^{i} R /{ }_{i} M$ is a finite group, the order of every element in ${ }^{i} R /{ }_{i} M$ is a divisor of $n^{2^{i}}$, and every prime number dividing the order of ${ }^{i} R /{ }_{i} M$ is a divisor of $n$.

It is now an immediate consequence of $(++)$ and $(+)$ that every group ${ }^{i} G /{ }_{i} N$ is finite, that its order is a divisor of the order of the group ${ }^{i} R{ }_{i} M$ and therefore every prime number dividing the order of ${ }^{i} \mathrm{G} /{ }_{i} N$ is a divisor of $n$, and that the order of every element in ${ }^{i} G /{ }_{i} N$ is a divisor of $n^{2^{i}}$. This completes the proof of Theorem 4 and of the following fact.

Corollary 3. If $G / N$, for $N$ a normal subgroup of the group $G$, is of finite order $n$, then the order of every element in ${ }^{i} G /{ }_{i} N$ is a divisor of $n^{2^{i}}$.

Remark 1 . That the preceding results have a somewhat limited applicability on general, not necessarily minimal, central chains may be seen from the following considerations. Suppose that $N_{0}, N_{1}, \cdots, N_{i}, \cdots$ is a central chain of the group $G$. Then $M=N_{0}$ is a normal subgroup of $G$, and the minimal central chain ${ }_{i} M$, determined by $M$, meets the following requirement: ${ }_{i} M \leqq N_{i}$ for every $i$, as may be seen by complete induction. Suppose now in addition that $G / M$ be finite. Then we deduce from Theorem 4 the finiteness of ${ }^{i} G /{ }_{i} M$. But ${ }_{i} M \leqq{ }^{i} G \cap N_{i}$ for every $i$. Hence ${ }^{i} G /\left({ }^{i} G \cap N_{i}\right)$ is finite too. We note the following interesting special case of this result.

If $N_{i}$ is a central chain of $G$ such that $G / N_{0}$ is finite, and such that $N_{i} \leqq{ }^{i} G$ for every $i$, then ${ }^{i} G / N_{i}$ is finite for every $i$.

Remark 2. It may be of interest to note that Corollary 4 of $\S 3$ is really a special case of Theorem 4. If we choose in particular $N=(G, G)$, then ${ }_{i} N={ }^{i+1} G$. Hence it follows from Theorem 4 that every ${ }^{i} G /{ }^{i+1} G$ is finite if $G /(G, G)$ is finite, and from this fact one deduces the Corollary $4^{\prime}$ of $\S 3$ 
which states that every $G /{ }^{i} G$ is finite if $G /(G, G)$ is finite. If now the hypotheses of Corollary 4 of $\S 3$ are satisfied by $G$ and its normal subgroup $N$, then it is readily seen that both $G / N$ and $G /(G, G)$ are finite, and hence we may deduce from Theorem 4 that both $G /{ }^{i} G$ and ${ }^{i} G /{ }_{i} N$ are finite for every $i$, a fact that is equivalent to the finiteness of every $G /{ }_{i} N$.

Corollary 4. If $N$ is a normal subgroup of the group $G$, and if $G / N$ is finite, then the following three properties imply each other.

(a) ${ }^{i} G /{ }_{i} N$ is, for every $i$, the subgroup of the elements of order different from 0 in $G /{ }_{i} N$.

(b) 1 is, for every $i$, the only element of order different from 0 in ${ }^{i-1} G /{ }^{i} G$.

(c) 1 is, for every $i$, the only element of order different from 0 in $G /{ }^{i} G$.

If the conditions (a) to (c) are satisfied, and if $i$ and $h$ are integers such that $0<h \leqq i+1$, then ${ }_{i} N /{ }_{i+h} N$ is the direct product of the abelian group $\left({ }_{i} N \cap{ }^{i+h} G\right) /{ }_{i+h} N$ without element of order 0 and of an abelian group all of whose elements, not 1 , are of order 0 .

Proof. That (a) implies (b) is obvious, and that (b) implies (c) is readily seen by complete induction with regard to $i$. That (c) implies (a) is an immediate consequence of Theorem 4 which states the finiteness of the group ${ }^{i} G /{ }_{i} N$.

If $0<h \leqq i+1$, then $\left({ }_{i} N,{ }_{i} N\right) \leqq\left({ }^{i} G,{ }_{i} N\right) \leqq{ }_{2 i+1} N \leqq{ }_{i+h} N$ is a consequence of equation (3). Hence ${ }_{i} N /{ }_{i+h} N$ is abelian. Since ${ }^{i+h} G /{ }_{i+h} N$ is finite, so is $\left({ }_{i} N \cap{ }^{i+h} G\right) /{ }_{i+h} N$. Since ${ }_{i} N /\left({ }_{i} N \cap{ }^{i+h} G\right)$ and ${ }_{i} N^{i+h} G /{ }^{i+h} G$ are isomorphic groups, and since the latter group is a subgroup of ${ }^{i} G /{ }^{i+h} G$, it follows from (c) that ${ }_{i} N /\left({ }_{i} N \cap{ }^{i+h} G\right)$ does not contain elements of order different from 0 excepting 1, and now our last contention is an immediate consequence of a well known theorem $\left({ }^{25}\right)$.

It should be noted that (b) is satisfied whenever $G$ is a free group $\left({ }^{26}\right)$.

TheOREM 5. If $G / N$, for $N$ a normal subgroup of the group $G$, is a group of class $k$, then ${ }^{i} G /{ }_{i} N$ is a group of class less than $1+k /(i+1)$. Thus ${ }^{i} G /{ }_{i} N$ is abelian whenever $k \leqq i+1$.

Proof. The hypothesis that $G / N$ be of class $k$ is equivalent to the inequality ${ }^{k} G \leqq N$, and from this inequality one deduces readily ${ }^{i+k} G \leqq{ }_{i} N$. Denote now by $h$ the greatest integer less than $1+k /(i+1)$. Then one deduces readily from formula (3) that

$$
{ }^{h}\left({ }^{i} G\right) \leqq(h+1) i+h G .
$$

But it follows from our choice of $h$ that

$$
(h+1) i+h=i+h(i+1) \geqq i+k,
$$

(25) See, for example, Baer [1].

(26) Magnus [2]. 
proving ${ }^{h}\left({ }^{i} G\right) \leqq{ }^{i+k} G \leqq{ }_{i} N$. Hence ${ }^{i} G /{ }_{i} N$ is of class $h$, as we intended to show. If in particular $k \leqq i+1$, then $h=1$ and ${ }^{i} G /{ }_{i} N$ is abelian.

Corollary 5. If $G / N$, for $N$ a normal subgroup of $G$, is a finite nilpotent group, then every ${ }^{i} G /{ }_{i} N$ is a finite nilpotent group.

This is an immediate consequence of the Theorems 4 and 5 , since a finite group is nilpotent if, and only if, it is of class $k$ for some positive integer $k$.

7. The $i$ th representation groups of $G / N$. If $N$ is a normal subgroup of the free group $G$, then ${ }^{i} G /{ }^{i+1} G$ is known $\left({ }^{27}\right)$ to be a free abelian group, and thus the subgroup $\left({ }_{i} N{ }^{i+1} G\right) /{ }^{i+1} G$ of ${ }^{i} G /{ }^{i+1} G$ is a free abelian group too $\left.{ }^{28}\right)$. But $\left({ }_{i} N^{i+1} G\right) /{ }^{i+1} G$ and ${ }_{i} N /\left({ }_{i} N \cap{ }^{i+1} G\right)$ are isomorphic groups. Consequently the abelian group ${ }_{i} N /{ }_{i+1} N$ is the direct product of $\left({ }_{i} N \cap{ }^{i+1} G\right) /{ }_{i+1} N$ and of a free abelian group. If the subgroup $W$ of $G$ contains ${ }_{i+1} N$, and if ${ }_{i} N /{ }_{i+1} N$ is the direct product of $\left({ }_{i} N \cap{ }^{i+1} G\right){ }_{i+1} N$ and of $W /{ }_{i+1} N$, then we are going to term ${ }^{i} G / W$ an ith representation group of $G / N$.

This definition may be justified by the following two reasons:

A. It is readily seen that the 0 th representation groups of $G / N$ coincide with the reduced free central extensions of $G / N$ which we considered in $\S 3$, and it has been pointed out in Remark 1 of $\$ 3$ that these are the same as I. Schur's "Darstellungsgruppen."

B. Every $i$ th representation group of $G / N$ is a central extension of $\left({ }_{i} N \cap{ }^{i+1} G\right) /{ }_{i+1} N$ by ${ }^{i} G /{ }_{i} N$, and these two groups are known $\left({ }^{29}\right)$ to be invariants of $G / N$. In the course of our investigation we shall have to consider groups of the form $G / W$ where ${ }_{i+1} N \leqq W$ and where ${ }_{i} N /{ }_{i+1} N$ is the direct product of $\left({ }_{i} N \cap{ }^{i+1} G\right) /{ }_{i+1} N$ and of $W /{ }_{i+1} N$, and these groups $G / W$ are extensions of an $i$ th representation group ${ }^{i} G / W$ by the group $G /{ }^{i} G$. But this latter group is certainly not an invariant of $G / N$.

In Remark 1 of $\$ 3$ we have pointed out that there may exist more than one 0 th representation group of $G / N$. It will be the object of this section to give a survey of the $i$ th representation groups of $G / N$.

We begin by generalizing the concept of splitting or trivial extension.

If $D$ is a subgroup of the group $E$, then the central factor set $f(x, y)$ of $E$ in the abelian group $Z$ shall be termed $D$-trivial, whenever

$$
f(x, y)=1 \text { for } x \text { or } y \text { in } D .
$$

Note that apart from exceptional cases, not every central transformation set of $E$ in $Z$ has the property (8). Thus it is impossible to expect that all the factor sets in a class of associate factor sets have property (8).

Theorem 1. If $D$ is a normal subgroup of the group $E$, then the following properties of the central extension $G$ of the group $Z$ by the group $E$ imply each other.

(27) Magnus [2].

(28) See footnote 9 above.

(29) See footnote 3 above. 
(a) $\left.{ }^{(30}\right)$ There exists a homomorphism of $G$ upon a central extension of $Z$ by $E / D$ which induces the identity in $Z$ and the natural homomorphisms of $E$ upon $E / D$.

(b) ${ }^{(11)}$ There exists a normal subgroup $R$ of $G$ such that $R Z / Z=D$ and $R \cap Z=1$.

(c) The central extension $G$ of $Z$ by $E$ may be determined by a $D$-trivial central factor set of $E$ in $Z$.

Proof. If the homomorphism $\eta$ of $G$ upon the central extension $H$ of $Z$ by $E / D$ induces the identity in $Z$ and the natural homomorphism of $E$ upon $E / D$, then the kernel $R$ of this homomorphism $\eta$ satisfies $R Z / Z=D$ and $R \cap Z=1$, showing that (b) is a consequence of (a).

Assume next the existence of a normal subgroup $R$ of $G$ such that $D=R Z / Z$ and $R \cap Z=1$. Then $E / D$ and $G / R Z$ are essentially the same groups. If $X$ is any coset of $E / D$, then let $r(X)$ be an element in the coset of $G / R Z$ which corresponds to $X$, in particular $r(1)=1$. If $x$ is any element in $E$ and $C(x)^{\prime}$ the coset of $G / Z$ which corresponds to $x$, then $C(x) r(D x)^{-1}$ is a coset of $G / Z$ which corresponds to an element in $D$ and which therefore contains a uniquely determined element $s(x)$ in $R$. Consequently $s(x) r(D x)$ is a representative of $C(x)$. The representative of $C(1)$ is 1 and the representative of $C(x)$ for $x$ in $D$ is the element $s(x)$ in $R$, since $r(1)=1$. Hence

$$
f(x, y)=s(x) r(D x) s(y) r(D y)[s(x y) r(D x y)]^{-1}
$$

is a central factor set of $E$ in $Z$ which defines the extension $G$. If $x$ belongs to $D$, then $D x y=D y$ and $r(D x)=r(1)=1$. Furthermore $C(x y) r(D x y)^{-1}$ $=C(x) C(y) r(D y)^{-1}$ contains $s(x) s(y)$, since $s(x)$ belongs to $C(x)$, and this implies $s(x y)=s(x) s(y)$, since a coset of $R Z / Z$ contains one and only one element in the subgroup $R$. From these facts we infer $f(x, y)=1$ for $x$ in $D$. If next $y$ belongs to $D$, then $r(D y)=1$ and $r(D x y)=r\left(D x y x^{-1} x\right)=r(D x)$, since $D$ is a normal subgroup of $E$. Consequently

$$
C(x y) r(D x y)^{-1}=\left[C(x) r(D x)^{-1}\right]\left[r(D x) C(y) r(D x)^{-1}\right] .
$$

The first factor on the right side of this equation contains the element $s(x)$ and the second factor the element $r(D x) s(y) r(D x)^{-1}$ which belongs to $R$, since $R$ is a normal subgroup of $G$. Since a coset of $R Z / Z$ contains one and only one element in $R$, it follows now that $s(x y)=s(x) r(D x) s(y) r(D x)^{-1}$, and this implies clearly $f(x, y)=1$ for $y$ in $D$. Thus we have shown that $f(x, y)$ is $D$-trivial, proving that (c) is a consequence of (b).

We precede the proof of the last implication by the proof of the following lemma.

(30) This property (a) implies that $G$ is $i$-splitting whenever $E / D$ is of class $i$.

( ${ }^{31}$ ) Condition (b) implies that $Z$ is a direct factor of the uniquely determined subgroup $S$ of $G$ which satisfies $Z \leqq S$ and $S / Z=D$. But from this property we could not infer the existence of a normal subgroup $R$ of $G$ such that $S$ is the direct product of $R$ and $Z$. 
(d) If the central factor set $f(x, y)$ of $E$ in $Z$ is $D$-trivial, then a central factor set $F(X, Y)$ of $E / D$ in $Z$ is defined by

$$
F(D x, D y)=f(x, y) \text { for } x, y \text { in } E .
$$

If $t$ is an element in $D$, then we deduce from the $D$-triviality of the central factor set $f(x, y)$ and from the fundamental identity (5) that

$$
f(t x, y)=f(t, x) f(t x, y)=f(x, y) f(t, x y)=f(x, y)
$$

and

$$
f(x, y t)=f(x, y t) f(y, t)=f(x, y) f(x y, t)=f(x, y) .
$$

Since $D$ is a normal subgroup of $E$, this implies $f(x, y)=f\left(x^{\prime}, y^{\prime}\right)$ whenever $x \equiv x^{\prime}$ and $y \equiv y^{\prime}$ modulo $D$. The definition given in (d) leads therefore to a single-valued function $F(X, Y)$ of its two variables. That $F(X, Y)$ is a central factor set of $E / D$ in $Z$ is now immediately obvious, completing the proof of the lemma $(\mathrm{d})$.

If $f(x, y)$ is a $D$-trivial central factor set of $E$ in $Z$, then it is possible to select a representative $r(x)$ in the coset of $G / Z$ corresponding to $x$ in $E$ such that $r(1)=1$ and $f(x, y)=r(x) r(y) r(x y)^{-1}$. From (d) we deduce the existence of a central extension $H$ of $Z$ by $E / D$ which is determined by $F(X, Y)$. There exists therefore a representative $v(X)$ of the coset of $H / Z$, corresponding to $X$ in $E / D$, such that $v(1)=1$ and $F(X, Y)=v(X) v(Y) v(X Y)^{-1}$. From $F(D x, D y)$ $=f(x, y)$ we deduce finally the existence of a uniquely determined homomorphism $\eta$ of $G$ upon $H$ which maps $r(x)$ upon $v(D x)$ and which leaves invariant the elements in $Z$. This homomorphism $\eta$ meets the requirements of (a). Thus (a) has been shown to be a consequence of (c), completing the proof of the theorem.

From the lemma (d) we infer that the $D$-trivial central factor set $f(x, y)$ is a centralized transformation set in the central extension of $Z$ by $E / D$ which is determined by the central factor set $F(X, Y)$. Thus we obtain the following result.

CoROLlaRY 1. If $D$ is a normal subgroup of the group $E$, and if the properties (a) to (c) are satisfied by the central extension $G$ of $Z$ by $E$, then there exists a central extension of $Z$ by $E / D$ which splits the extension $G$ of $Z$ by $E$.

We cannot expect the converse of this statement to be true in general. To derive a criterion for its validity the following concept will be needed, which seems to be of interest for its own sake. We term $G / N$, for $N$ a normal subgroup of $G$, a fully invariant quotient group of $G$ if $N$ is mapped upon 1 by every homomorphism of $G$ into $G / N$.

If $N$ is a word subgroup $\left({ }^{32}\right)$ of $G$, then $G / N$ is a fully invariant quotient group of $G$.

(s2) Neumann [1, Definition 6.1, p. 510]. 
Proof. If $x$ is an element in the word subgroup $N$ of $G$, then there exists a "word" $w\left(x_{1}, \cdots, x_{n}\right)$ in the $n$ variables $x_{i}$ (and their inverses) with the following properties: (i) If $u_{1}, \cdots, u_{n}$ are elements in $G$, then $w\left(u_{1}, \cdots, u_{n}\right)$ belongs to $N$; (ii) there exist elements $y_{1}, \cdots, y_{n}$ in $G$ such that $x=w\left(y_{1}, \cdots, y_{n}\right)$. If $\eta$ is a homomorphism of $G$ into $G / N$, then $x^{\eta}=w\left(y_{1}^{\eta}, \cdots, y_{n}^{\eta}\right)$. There exist elements $z_{i}$ in $G$ such that $y_{i}^{\eta}=N z_{i}$, and hence we have $x^{\eta}=w\left(N z_{1}, \cdots, N z_{n}\right)$ $\equiv w\left(z_{1}, \cdots, z_{n}\right)$ modulo $N$. But it is a consequence of (i) that $w\left(z_{1}, \cdots, z_{n}\right)$ belongs to $N$, and thus we have shown $x^{\eta} \equiv 1$ modulo $N$ for every $x$ in $N$ and every homomorphism $\eta$ of $G$ into $G / N$, so that $G / N$ has been shown to be a fully invariant quotient group of $G$.

The following special case of this criterion is most important for our applications.

$G /{ }^{i} G$ is a fully invariant quotient group of $G$.

The word subgroups, however, are not the only subgroups leading to fully invariant quotient groups. If $G$ is, for instance, the direct product of an infinite cyclic group $Z$ and a finite cyclic group $F$, then $F$ is not a word subgroup of $G$, though $G / F$ is a fully invariant quotient group of $G$. In this respect at least the following fact may be proven.

If $G / N$ is a fully invariant quotient group of $G$, then $N$ is a fully invariant subgroup ${ }^{(33)}$ of $G$.

Proof. If $\eta$ is an endomorphism of $G$, then mapping the element $x$ in $G$ upon the coset $N x^{\eta}$ of $G / N$ constitutes a homomorphism $\kappa$ of $G$ in to $G / N$. Consequently $N=N^{\star}=N N^{\eta}$, proving $N^{\eta} \leqq N$, as we intended to show.

If $n$ is a number greater than 1 , and if $G_{n}$ is the subgroup of $G$ which is generated by those elements in $G$ whose order is a divisor of $n$, then $G_{n}$ is certainly a fully invariant subgroup of $G$. But it is easy to construct abelian groups $G$ such that $G / G_{n}$, for suitable $n$, is not a fully invariant quotient group of $G$. For instance let $G$ be the direct product of a cyclic group $U$ of order $p$ by a cyclic group $V$ of order $p^{2}$. Then there exists a homomorphism $\nu$ of $G$ upon $G / G_{p}$ which maps $V$ upon 1 and which maps $U$ isomorphically upon $G / G_{p}$.

Corollary 1'. If the quotient group $E / D$ is fully invariant, and if there exists a central extension of $Z$ by $E / D$ which splits the central extension $G$ of $Z$ by $E$, then conditions (a) to (c) of Theorem 1 are satisfied by the extension $G$ of $Z$ by $E$.

Proof. There exists a central extension $H$ of $Z$ by $E / D$ which splits the central extension $G$ of $Z$ by $E$. Consequently there exists a group $K$ with the following properties:

(i) $K=G H$ and $Z=G \cap H$.

(ii) Every element in $G$ permutes with every element in $H$. $[4,5]$.

(33) This concept is due to F. Levi. For a discussion of this and related concepts, see Baer 
(iii) There exists a subgroup $S$ of $K$ such that $K=S H$ and $1=S \cap H$. Clearly $K$ is a centralizer extension of $H$ by $E$ and a centralizer extension of $G$ by $E / D$.

In the coset of $K / H$ which corresponds to the element $x$ in $E$ there exists one and only one element $s(x)$ in $S$. Since $S$ is a subgroup of $K$, we obtain an isomorphism of $E$ upon $S$ by mapping $x$ upon $s(x)$. Since $K / G$ and $E / D$ are essentially the same, mapping $x$ in $E$ upon $G s(x)$ constitutes a homomorphism of $E$ into $E / D$. But the quotient group $E / D$ is fully invariant, and hence $D$ is mapped upon 1 by this homomorphism, implying that $s(x)$ is in $G$ whenever $x$ is in $D$. Put $R=s(D)$. Then $R$ is a subgroup of both $S$ and $G$, and clearly $R Z / Z=D$. Since $D$ is a normal subgroup of $E$, it follows that $s(D)=R$ is a normal subgroup of $s(E)=S$. If $y$ is any element in $K$, then $y=y^{\prime} y^{\prime \prime}$ for $y^{\prime}$ in $S$ and $y^{\prime \prime}$ in $H$. Since $R$ is a normal subgroup of $S$, we have $y^{\prime-1} R y^{\prime}=R$, and since $R$ is a subgroup of $G$, we infer $y^{\prime \prime-1} R y^{\prime \prime}=R$ from (ii). Consequently $y^{-1} R y=R$, proving that $R$ is a normal subgroup of $K$. Thus $R$ is a normal subgroup of $G$ too. Finally $R \cap Z=1$ is a consequence of $S \cap H=1$. Thus condition (b) of Theorem 1 has been shown to be a consequence of our hypotheses, and this completes the proof.

If $D$ is a normal subgroup of the group $E$, then we term the central extension $G$ of $Z$ by $E$ a $D$-trivial extension, provided it satisfies the conditions (a) to (c) of Theorem 1 . It is an immediate consequence of property (c) that the $D$-trivial extensions form a subgroup $\operatorname{Ext}(Z, E ; D)$ of the group $\operatorname{Ext}(Z, E)$ of all the central extensions of $Z$ by $E$. If in particular $D$ happens to be of finite order $n$, then we may deduce from Theorem 3 in $\$ 6$ and from its proof that $\operatorname{Ext}(Z, E)^{n} \leqq \operatorname{Ext}(Z, E ; D)$.

If $F(X, Y)$ is a central factor set of $E / D$ in $Z$, then a $D$-trivial central factor set of $E$ in $Z$ is defined by the equation

$$
f(x, y)=F(D x, D y) \text { for } x, y \text { in } E .
$$

It is readily seen that $f(x, y)$ is a central transformation set of $E$ in $Z$ if. $F(X, Y)$ is a central transformation set of $E / D$ in $Z$, and the lemma (d), proved during the proof of Theorem 1 , shows that every $D$-trivial central factor set of $E$ in $Z$ may be obtained in this fashion. Thus we have obtained a homomorphism of $\operatorname{Ext}(Z, E / D)$ upon (the full group) $\operatorname{Ext}(Z, E ; D)$, and we shall refer to this homomorphism as to the natural homomorphism of the group $\operatorname{Ext}(Z, E / D)$ upon the group $\operatorname{Ext}(Z, E ; D)$.

THEOREM 2. If $D$ is a normal subgroup of the group $E$, then the following properties of the central extension $H$ of $Z$ by $E / D$ imply each other.

(i) The extension $H$ belongs to the kernel of the natural homomorphism of $\operatorname{Ext}(Z, E / D)$ upon $\operatorname{Ext}(Z, E ; D)$.

(ii) There exists a homomorphism of $E$ into $H$ which induces the identity in $E / D$. 
(iii) If $d(x, y)$ is a central factor set of $E / D$ in $D /(E, D)$ which defines the central extension $E /(D, E)$ of $D /(D, E)$ by $E / D$, then there exists a homomorphism $\eta$ of $D /(D, E)$ into $Z$ such that $d(x, y)^{\eta}$ defines the central extension $H$ of $Z$ by $E / D$.

Proof. The extension $H$ of $Z$ by $E / D$ is mapped by the natural homomorphism of $\operatorname{Ext}(Z, E / D)$ upon $\operatorname{Ext}(Z, E ; D)$ upon a certain $D$-trivial central extension $G$ of $Z$ by $E$, and if the extension $H$ of $Z$ is determined by the central factor set $F(X, Y)$ of $E / D$ in $Z$, then the extension $G$ is determined by the $D$-trivial central factor set $f(x, y)=F(D x, D y)$ of $E$ in $Z$. It is easy to see that there exists a homomorphism $\gamma$ of $G$ upon $H$ which induces the identity in $Z$ and which induees the natural homomorphism of $E$ upon $E / D$ (see Theorem 1).

If (i) holds true, then $G$ is a splitting central extension of $Z$ by $E$, and there exists therefore a subgroup $S$ of $G$, isomorphic to $E$, such that $G$ is the direct product of $Z$ and $S$. If $x$ is an element in $E$, then there exists one and only one element $s(x)$ in the cross cut of $S$ and of the coset of $G / Z$ which corresponds to $x$. Mapping $x$ upon $s(x)$ constitutes an isomorphism of $E$ upon $S$, and mapping $x$ upon $s(x)^{r}$ constitutes a homomorphism of $E$ into $H$ which induces the natural homomorphism of $E$ upon $E / D$ and which therefore induces the identity in $E / D$. Thus (ii) is a consequence of (i).

If (ii) holds true, then there exists a homomorphism $\kappa$ of $E$ into $H$ such that $Z x^{\kappa}$ is the coset of $H / Z$ which corresponds to the coset $D x$ of $E / D$. Thus we have in particular $H=Z E^{\kappa}$ and $D^{\star}=Z \cap E^{\star}$. Hence $(E, D)^{\star} \leqq(H, Z)$ $=1$ and $\kappa$ induces therefore a homomorphism $\eta$ of $D /(E, D)$ into $Z$. It is now readily verified that $\eta$ meets all the requirements of (iii), showing that (iii) is a consequence of (ii).

If (iii) holds true, then there exists in the coset of $[E /(D, E)] /[D /(D, E)]$ which corresponds to the element $X$ in $E / D$ an element $r(X)$ such that $r(1)=1$ and $d(X, Y)=r(X) r(Y) r(X Y)^{-1}$. Every element $x$ in $E /(D, E)$ may be written uniquely in the form $x=x^{\prime} r\left(x^{\prime \prime}\right)$ where $x^{\prime}$ is in $D /(D, E)$ and $x^{\prime \prime}=[D /(D, E)] x$. If $x$ and $y$ are both in $E /(D, E)$, then

or

$$
x^{\prime} y^{\prime} r\left(x^{\prime \prime}\right) r\left(y^{\prime \prime}\right)=x^{\prime} r\left(x^{\prime \prime}\right) y^{\prime} r\left(y^{\prime \prime}\right)=x y=(x y)^{\prime} r\left((x y)^{\prime \prime}\right)
$$

$$
d\left(x^{\prime \prime}, y^{\prime \prime}\right)=r\left(x^{\prime \prime}\right) r\left(y^{\prime \prime}\right) r\left((x y)^{\prime \prime}\right)^{-1}=y^{\prime-1} x^{\prime-1}(x y)^{\prime} .
$$

If the homomorphism $\eta$ of $D /(D, E)$ into $Z$ meets the requirements of (iii), then let

$$
t(x)=\left([(D, E) x]^{\prime-1}\right)^{\eta}
$$

for $x$ in $E$, and it is readily seen that

$$
t(x) t(y) t(x y)^{-1}=d(D x, D y)^{\eta} .
$$

Since $t(x)$ is in $Z$ and $t(1)=1, Y$ is a splitting extension of $Z$ by $E$, and (i) is therefore a consequence of (iii), completing the proof of this theorem. 
Remark 1. Applying (iii) of Theorem 2 we see that the natural homomorphism of $\operatorname{Ext}(Z, E / D)$ upon $\operatorname{Ext}(Z, E ; D)$ is an isomorphism if, and only if, every homomorphism of $D /(D, E)$ into $Z$ maps $d(x, y)$ upon a central transformation set of $E / D$ in $Z$. This is the case if in particular every homomorphism of $D /(D, E)$ into $Z$ maps $D /(D, E)$ upon 1 , and this will be the case, for example, if $D /(D, E)$ and $Z$ are finite groups of relatively prime orders and so on.

Remark 2. If $d(x, y)$ happens to be the free central factor set of $E / D$, then it may be shown that there exists to every central factor set of $E / D$ in $Z$ a homomorphism of $D /(D, E)$ which maps $d(x, y)$ upon the given factor set. In this case we may deduce, therefore, from (iii) that the natural homomorphism maps $\operatorname{Ext}(Z, E / D)$ upon 1 and that consequently the only $D$-trivial extension of $Z$ by $E$ is the splitting extension of $Z$ by $E$, that is, their direct product.

The consequence of Theorem 2 which will be most important for our applications is contained in the following fact.

Corollary 2. The central extension $H$ of $Z$ by $E /{ }^{i} E$ is splitting if, and only if, it is of class $i$ and belongs to the kernel of the natural homomorphism of $\operatorname{Ext}\left(Z, E /{ }^{i} E\right)$ upon $\operatorname{Ext}\left(Z, E ;{ }^{i} E\right)$.

Proof. The necessity of the conditions is an almost immediate consequence of Theorem 1 of $\S 6$. Thus we assume conversely that the conditions are satisfied by the central extension $H$ of $Z$ by $E /{ }^{i} E$. Then there exists by (ii) of Theorem 2 a homomorphism $\alpha$ of $E$ into $H$ which induces the identity in $E /{ }^{i} E$. Since $H$ is of class $i$, we have ${ }^{i} H=1$, and thus ${ }^{i} E^{\alpha}=1$ is a consequence of ${ }^{i} E^{\alpha} \leqq i H$. This shows that $E^{\alpha}$ is a subgroup of $H$ with the following properties, considering that $\alpha$ induces an isomorphism between $E /{ }^{i} E$ and $H / Z$ : $H=Z E^{\alpha}$ and $1={ }^{i} E^{\alpha}=Z \cap E^{\alpha}$. Hence $H$ is the direct product of $Z$ and $E^{\alpha}$, since $Z$ is part of the center of $H$, completing the proof.

The central factor set $f(x, y)$ of the group $E$ in the abelian group $Z$ may be termed of class $i$ if the central extension of $Z$ by $E$ which is determined by $f(x, y)$ is of class $i$.

Lemma 1. The central factor set $f(x, y)$ of $E$ in $Z$ is of class $i$ if, and only if, $E$ is of class $i$ and $f(x, y)=f(y, x)$ for $x$ in ${ }^{i-1} E$.

Proof. Denote by $G$ the central extension of $Z$ by $E$ which is determined by $f(x, y)$. Then it is easily seen that ${ }^{i} E={ }^{i} G Z / Z$.

If $f(x, y)$ is of class $i$, then $G$ is of class $i$ or ${ }^{i} G=1$. This implies ${ }^{i} E=1$ so that $E$ is of class $i$. Furthermore we deduce from ${ }^{i} G=1$ that ${ }^{i-1} G Z$ is part of the center of $G$. Thus if $r(x)$ is a representative of the coset of $G / Z$ which corresponds to $x$ such that $r(1)=1, f(x, y)=r(x) r(y) r(x y)^{-1}$, then $r(x)$ is in the center of $G$ and $x$ in the center of $E$, provided $x$ belongs to ${ }^{i-1} E$, and thus $f(x, y)=f(y, x)$ whenever $x$ is in ${ }^{i-1} E$. 
If conversely the conditions are satisfied, then it is obvious that ${ }^{i-1} G Z$ is part of the center of $G$, implying ${ }^{i} G=1$, as we intended to show.

LemMa 2. If $G$ is the central extension of $Z$ by $E$ which is determined by the central factor set $f(x, y)$, if $N$ is a normal subgroup of $G$ and $D=N Z / Z$, and if the homomorphism $\eta$ of $Z$ into $Z \cap N$ maps $Z \cap N$ upon 1 , then $f(x, y)^{n}$ is associate to a D-trivial central factor set of $E$ in $Z \cap N$.

Proof. We deduce from Lemma 2 of $\$ 2$ the existence of a homomorphism $\kappa$ of $G$ upon a central extension $H$ of $N \cap Z$ by $E$ with the properties: $\kappa$ induces $\eta$ in $Z$ and the identity in $E$. Since $N$ is a normal subgroup of $G$, and since $H$ is the image of $G$ under $\kappa, N^{\star}$ is a normal subgroup of $H$. The cross cut of $N^{\star}=M$ and $Z \cap N$ is 1 , since $Z \cap N$ is mapped by $\eta$ upon 1 , and since elements, not in $Z$, are not mapped by $\kappa$ upon elements in $Z \cap N$. Clearly $D=M[Z \cap N]$. Thus we see that condition (b) of Theorem 1 is satisfied by the central extension $H$ of $Z \cap N$ by $E$ which is determined by $f(x, y)^{\eta}$, and our contention is a consequence of (c) of Theorem 1.

Theorem 3. If the central extension $G$ of the group $Z$ by the group $E$ is determined by the central factor set $f(x, y)$, then the following properties of the homomorphism $\eta$ of $Z$ into $Z \cap{ }^{i} G$ are equivalent.

(a) $\left(Z \cap{ }^{i} G\right)^{\eta}=1$.

(b) $f(x, y)^{\eta}$ is associate to an ${ }^{i} E$-trivial central factor set $g(x, y)$ of $E$ in $Z \cap{ }^{i} G$, satisfying $g(x, y)=g(y, x)$ for $x$ in ${ }^{i-1} E$.

Proof. We deduce from Lemma 2 of $\$ 2$ the existence of a homomorphism $\kappa$ of $G$ upon a central extension $H$ of $Z \cap{ }^{i} G$ by $E$ with the properties : $\kappa$ induces $\eta$ in $Z$ and the identity in $E$.

If condition (a) is satisfied by $\eta$, then we deduce from Lemma 2 that $f(x, y)^{\eta}$ is associate to an ${ }^{i} E$-trivial central factor set $g(x, y)$ of $E$ in $Z \cap{ }^{i} G$, since ${ }^{i} E={ }^{i} G Z / Z$. A homomorphism $\gamma$ of $G$ upon $H /{ }^{i} H$ is defined by mapping the element $v$ in $G$ upon ${ }^{i} H v^{\kappa}$. From $\left({ }^{34}\right){ }^{i} H \cap{ }^{i} G \cap Z=1$ we infer that $\kappa$ and $\gamma$ induce in $Z$ the same homomorphism $\eta$, and the central extension $H /{ }^{i} H$ of $Z \cap{ }^{i} G$ by $E /{ }^{i} E$ is determined by a central factor set $h(x, y)$ which may be defined by the equation $h\left({ }^{i} E x,{ }^{i} E y\right)=g(x, y)$. But $H /{ }^{i} H$ is of class $i$, and hence we deduce $h(x, y)=h(y, x)$ for $x$ in ${ }^{i-1} E /{ }^{i} E$ from Lemma 1 . Consequently $g(x, y)=g(y, x)$ for $x$ in ${ }^{i-1} E$. Thus (b) is a consequence of (a).

Suppose conversely that (b) is satisfied. If we define now $h(x, y)$ by the equation $h\left({ }^{i} E x,{ }^{i} E y\right)=g(x, y)$, then it follows from Lemma 1 and from the lemma (d) obtained in the course of the proof of Theorem 1 that $h(x, y)$ is a central factor set of $E /{ }^{i} E$ in $Z \cap{ }^{i} G$ and that this factor set is of class $i$. From

(34) This equation may be verified as follows: If $x$ is an element in ${ }^{i} H \cap{ }^{i} G \cap Z$, then $x=y^{k}$ for $y$ in ${ }^{i} G$. But $y^{k}$ belongs to ${ }^{i} G \cap Z$ and hence $y$ belongs to $Z$. Applying (a) we infer that $x=y^{k}=1$, as we intended to show. 
these facts one readily deduces that $g(x, y)$, and therefore $f(x, y) \eta$, determines an $i$-splitting central extension of $Z^{\eta} \leqq Z \cap{ }^{i} G$ by $E$. But then condition (a) is an immediate consequence of Theorem 2 of $\$ 6$, completing the proof of the equivalence of (a) and (b).

Corollary 3. If $N$ is a normal subgroup of the free group $G$, if the central extension $H=G /{ }_{i+1} N$, for $0 \leqq i$, of $M={ }_{i} N /{ }_{i+1} N$ by $E=G /{ }_{i} N$ is determined by the central factor set $f(x, y)$ of $E$ in $M$, then the following properties of the central factor set $g(x, y)$ of $E$ in $M \cap^{i+1} H$ imply each other.

(a) There exists a homomorphism $\eta$ of $M$ into $M \cap^{i+1} H$ such that $\left(M \cap^{i+1} H\right)^{\text {n }}$ $=1$ and such that $f(x, y)^{n}$ and $g(x, y)$ are associate central factor sets of $E$ in $M \cap{ }^{i+1} H$.

(b) $g(x, y)$ is associate to an ${ }^{i+1} E$-trivial central factor $h(x, y)$ of $E$ in $M \cap{ }^{i+1} H$ such that $h(x, y)=h(y, x)$ for $x$ in ${ }^{i} E$.

Proof. If condition (a) is satisfied, then we deduce from Theorem 3 the existence of an ${ }^{i+1} E$-trivial central factor set $h(x, y)$ of $E$ in $M \cap^{i+1} H$, satisfying $h(x, y)=h(y, x)$ for $x$ in ${ }^{i} E$, such that $h(x, y)$ and $f(x, y)^{n}$ are associate. But the latter factor set and $g(x, y)$ are associate, showing that (b) is a consequence of (a).

Suppose next that (b) is satisfied by $g(x, y)$. There exists a central extension $V$ of $Z=M \cap^{i+1} H$ by $E$ which realizes the central factor set $g(x, y)$ of $E$ in $Z$. It is not difficult to construct a homomorphism $\alpha$ of the free group $G$ into $V$ which induces the identity in $E$. Then ${ }_{i} N^{\alpha} \leqq Z$, and ${ }_{i+1} N^{\alpha}=1$ is a consequence of the fact that $Z$ is part of the center of $V$. Thus a homomorphism $\beta$ of $H$ into $V$ is induced by $\alpha$. Clearly $\beta$ induces the identity in $E$ and satisfies $V=Z H^{\beta}$ and $Z \cap H^{\beta}=M^{\beta}$. Consequently $h(x, y), g(x, y)$ and $f(x, y)^{\beta}$ are associate central factor sets of $E$ in $Z=M \cap{ }^{i+1} H$. Applying Theorem 3 we find that $M \cap{ }^{i+1} H$ is mapped upon 1 by $\beta$, and thus we have shown that (a) is a consequence of $(b)$.

CoRollary 4. If the central extension $H$ of the group $M$ by the group $E$ is determined as in Corollary 3, then the following properties of the central extension $V$ of the group $M \cap^{i+1} H$ by $E$ imply each other:

(a) There exists a homomorphism of $H$ into $V$ which induces the identity in $E$ and which maps $M \cap^{i+1} H$ upon 1.

(b) There exists a central extension of class $i+1$ of $M \cap^{i+1} H$ by $E /{ }^{i+1} E$ which splits the extension $V$ of $M \cap^{i+1} H$ by $E$.

Proof. We denote by $h(x, y)$ a central factor set of $E$ in $M$ which determines the extension $H$ of $M$ by $E$, and by $v(x, y)$ a central factor set of $E$ in $M \cap{ }^{i+1} H$ which determines the extension $V$.

Assume now the existence of a homomorphism $\alpha$ of $H$ into $V$ which induces the identity in $E$ and which maps $M \cap^{i+1} H$ upon 1 . Then $M^{\alpha} \leqq M \cap^{i+1} H$, and $v(x, y)$ and $h(x, y)^{\alpha}$ are associate central factor sets of $E$ in $M \cap^{i+1} H$. 
Hence we infer from Corollary 3 the existence of an ${ }^{i+1} E$-trivial central factor set $w(x, y)$ of $E$ in $M \cap^{i+1} H$ which is associate to $v(x, y)$ and which satisfies $w(x, y)=w(y, x)$ for $x$ in ${ }^{i} E$. In the course of the proof of Theorem 1 we verified a lemma (d) from which we deduce the following fact: a central factor set $W(X, Y)$ of $E /^{i+1} E$ in $M \cap^{i+1} H$ is defined by $W\left(^{i+1} E x,{ }^{i+1} E y\right)=w(x, y)$.

If $X$ is in ${ }^{i}\left(E /{ }^{i+1} E\right)={ }^{i} E /{ }^{i+1} E$, then $W(X, Y)=W(Y, X)$, and thus it follows from Lemma 1 that $W(X, Y)$ is of class $i+1$. Denote now by $S$ the central extension of $M \cap^{i+1} H$ by $E /{ }^{i+1} E$ which is determined by $W(X, Y)$. Then $S$ is of class $i+1$, and it is clear that $w(x, y)$ is a centralizer transformation set of $E$ in $S$. But the central extension $V$ of $M \cap^{i+1} H$ by $E$ is determined by the central factor set $w(x, y)$, and hence $S$ splits $V$. Thus (b) is a consequence of $(a)$.

If (b) is satisfied by $V$, then we may apply Corollary $1^{\prime}$ since the quotient group $E /{ }^{i+1} E$ is fully invariant, as has been pointed out before. Hence we infer from Corollary $1^{\prime \prime}$ and Theorem 1 that $v(x, y)$ is associate to an ${ }^{i+1} E$ trivial central factor set $t(x, y)$ of $E$ in $M \cap^{i+1} H$. If $T$ is a central extension of $M \cap^{i+1} H$ by $E /{ }^{i+1} E$ which is of class $i+1$ and which splits the extension $V$, then there exist elements $t(x)$ in $T$ such that $t(1)=1$ and $t(x, y)=t(x) t(y) t(x y)^{-1}$. Since the central extension $V$ is defined by $t(x, y)$, there exists corresponding to every element $x$ in $E$ an element $r(x)$ in the corresponding coset of $V /\left(M \cap{ }^{i+1} H\right)$ such that $r(1)=1$ and $t(x, y)=r(x) r(y) r(x y)^{-1}$. Let $s(x)$ $=t(x)^{-1} r(x)$. Then $s(x)$ belongs to the coset of $V T / T$ which corresponds to $x$. Since $r(x)$ and $t(y)$ permute, it follows that $s(x y)=s(x) s(y)$ and that therefore the elements $s(x)$ form a representative subgroup $R$ of $V T$ modulo $T$. In particular $R \cap T=1$. Applying Theorem 1 of $\S 6$ we deduce ${ }^{i+1}(V T)$ ${ }^{i+1} R$ so that the cross cut of ${ }^{i+1} V$ and $T$ as well as the cross cut of ${ }^{i+1} R$ and $T$ is 1 . If $x$ is in ${ }^{i} E$, then $t(x, y) t(y, x)^{-1}$ is readily seen to belong to ${ }^{i+1} V$ as well as to $M \cap{ }^{i+1} H \leqq T$, showing that $t(x, y)=t(y, x)$ for $x$ in ${ }^{i} E$. Now we may infer from Corollary 3 the existence of a homomorphism $\beta$ of $M$ into $M \cap{ }^{i+1} H$ which maps $M \cap^{i+1} H$ upon 1 such that $h(x, y)^{\beta}$ and $v(x, y)$ are associate, and from this fact one readily deduces that (a) is a consequence of (b).

As in Corollary 3 we are going to assume throughout the remainder of this section that $N$ is a normal subgroup of the free group $G$, that $0 \leqq i$, and that

$$
H=G /{ }_{i+1} N, M={ }_{i} N /{ }_{i+1} N, E=G /{ }_{i} N \text { and } Z=M \cap{ }^{i+1} H .
$$

Denote by $\Xi$ the group $\operatorname{Ext}\left(Z, E /{ }^{i+1} E\right)$ of all the central extensions of $Z$ by $E /{ }^{i+1} E$, and by $\Xi^{*}=\operatorname{Ext}^{*}\left(Z, E /{ }^{i+1} E\right)$ the set of those central extensions $P$ of $Z$ by $E /{ }^{i+1} E$ which meet the requirement:

$\left(^{*}\right) P$ is a group of class $i+1$.

Denote by $\Delta$ the group $\operatorname{Ext}(Z, E)$ of all the central extensions of $Z$ by $E$, and by $\Delta^{+}=\operatorname{Ext}^{+}(Z, E)$ the set of all the central extensions $R$ of $Z$ by $E$ with the following property: 
(t) There exists a central extension of $Z$ by $E /{ }^{i+1} E$ which is of class $i+1$ and which splits the extension $R$ of $Z$.

Note that all the extensions in $\Delta^{+}$are $i+1$-splitting.

Finally the reader ought to remember the natural homomorphism of $\Xi$ upon the group $\operatorname{Ext}\left(Z, E ;{ }^{i+1} E\right)$ of all the ${ }^{i+1} E$-trivial central extensions of $Z$ by $E$ which we introduced just before stating Theorem 2 .

THEOREM 4. $\Xi^{*}$ is a subgroup of $\Xi$, and the natural homomorphism of $\Xi$ into $\Delta$ induces an isomorphism of $\Xi^{*}$ upon $\Delta^{+}$.

Proof. That $\Xi^{*}$ is a subgroup of $\Xi$ is a consequence of Lemma 1 , since $E /{ }^{i+1} E$ is of class $i+1$. That the natural homomorphism of $\Xi$ into $\Delta$ induces an isomorphism in $\Xi^{*}$ is an immediate consequence of Corollary 2. To show that $\Delta^{+}$is the image of $\Xi^{*}$ under the natural homomorphism we proceed as follows. Denote by $h(x, y)$ a central factor set of $E$ in $M$ which determines the central extension $H$ of $M$ by $E$, and denote by $R$ a central extension of $Z$ by $E$ which is determined by a central factor set $r(x, y)$ of $E$ in $Z$. It is a consequence of Corollary 4 that $R$ meets the requirement $(+)$ if, and only if, there exists a homomorphism $\alpha$ of $H$ into $R$ which induces the identity in $E$ and which maps $Z$ upon 1. The existence of such a homomorphism $\alpha$ of $H$ is clearly equivalent to the existence of a homomorphism $\beta$ of $M$ into $Z$ such that $Z^{\beta}=1$ and such that $r(x, y)$ and $h(x, y)^{\beta}$ are associate central factor sets of $E$ in $Z$. It is a consequence of Corollary 3 that the existence of such a homomorphism $\beta$ is equivalent to the existence of an ${ }^{i+1} E$-trivial central factor set $s(x, y)$ of $E$ in $Z$ which is associate to $r(x, y)$ and which satisfies $s(x, y)=s(y, x)$ for $x$ in ${ }^{i} E$. But it is a consequence of Lemma 1 and of the lemma (d) (which we obtained in the course of the proof of Theorem 1) that the existence of such a central factor set $s(x, y)$ is equivalent to the property: $R$ belongs to the image of $\Xi^{*}$ under the natural homomorphism of $\Xi$; this completes the proof.

Denote by $\Delta^{\circ}$ the set $\operatorname{Ext}^{\circ}(Z, E)$ of all the central extensions $Q$ of $Z$ by $E$ with the following property.

(o) There exists a homomorphism $\eta$ of $H$ upon $Q$ which maps $M$ upon $Z$ and which leaves invariant all the elements in $E$ and in $Z$.

Corollary 5. $\Delta^{\circ}$ is exactly a coset of $\Delta / \Delta^{+}$.

Proof. If the central extension $Q$ of $Z$ by $E$ is determined by the central factor set $q(x, y)$, and if the central extension $H$ of $M$ by $E$ is determined by the central factor set $h(x, y)$, then condition (0) is readily seen to be equivalent to the existence of a homomorphism $\kappa$ of $M$ upon $Z$ which leaves all the elements in $Z$ invariant and which maps $h(x, y)$ upon the central factor set $h(x, y)^{\kappa}$ associate to $q(x, y)$. The existence of elements in $\Delta^{\circ}$ is an immediate consequence of the fact that $Z$ is a direct factor of $M$, since-as has been pointed out before- $M / Z$ and ${ }_{i} N /\left({ }_{i} N \cap{ }^{i+1} G\right)$ are free abelian groups. The 
proof of Corollary 5 is now readily completed if one remembers the fact, obtained in the course of the proof of Theorem 4, that a central factor set $r(x, y)$ of $E$ in $Z$ determines an extension in $\Delta^{+}$if, and only if, there exists a homomorphism $\beta$ of $M$ into $Z$ such that $Z^{\beta}=1$ and such that $h(x, y)^{\beta}$ and $r(x, y)$ are associate.

If $C$ is a central extension of the abelian group $A$ by the group $B$, and if $D$ is a subgroup of $B$, then there exists one and only one subgroup $S$ of $C$ such that $A \leqq S$ and $S / A=D$. Clearly $S$ is a central extension of $A$ by $D$, and in this fashion we obtain a homomorphism of $\operatorname{Ext}(A, B)$ into $\operatorname{Ext}(A, D)$ which may be termed the natural homomorphism of $\operatorname{Ext}(A, B)$ into $\operatorname{Ext}(A, D)$. We note that the central extension $C$ of $A$ by $B$ belongs to the kernel of this natural homomorphism if, and only if, $A$ is a direct factor of the uniquely determined subgroup $S$ of $C$ which satisfies $A \leqq S$ and $S / A=D$.

We denote by $\Xi^{* *}=\operatorname{Ext}^{* *}\left(Z, E /{ }^{i+1} E\right)$ the set of all those central extensions $P$ of $Z$ by $E /{ }^{i+1} E$ which meet the following two requirements.

(*) $P$ is a group of class $i+1$.

$(* *) Z$ is a direct factor of $i P Z$.

Clearly $\left({ }^{i} P Z\right) / Z={ }^{i} E /{ }^{i+1} E$. Thus the kernel of the natural homomorphism of $\operatorname{Ext}\left(Z, E /{ }^{i+1} E\right)$ into $\operatorname{Ext}\left(Z,{ }^{i} E /{ }^{i+1} E\right)$ consists exactly of those central extensions $P$ of $Z$ by $E{ }^{i+1} E$ which meet the requirement $\left(^{* *}\right)$. Hence $\Xi^{* *}$ is exactly the cross cut of this kernel and of the group $\Xi^{*}$.

Theorem 5. $\Xi^{*} / \Xi^{* *}$ is essentially the same as the group $\operatorname{Abext}\left(Z,{ }^{i} E /{ }^{i+1} E\right)$ of all the abelian extensions of $Z$ by ${ }^{i} E /{ }^{i+1} E$.

Proof. If the central extension $P$ of $Z$ by $E /{ }^{i+1} E$ belongs to $Z^{*}$, then $P$ is of class $i+1$. Hence ${ }^{i+1} P=1$ so that ${ }^{i} P Z$ is part of the center of $P$ and is, therefore, abelian. But $P$ is mapped by the natural homomorphism of $\operatorname{Ext}\left(Z, E /{ }^{i+1} E\right)$ into $\operatorname{Ext}\left(Z,{ }^{i} E /{ }^{i+1} E\right)$ upon the central extension ${ }^{i} P Z$ of $Z$ by ${ }^{i} E /{ }^{i+1} E$, and thus we have shown that this natural homomorphism maps $\Xi^{*}$ into $A=\operatorname{Abext}\left(Z,{ }^{i} E /{ }^{i+1} E\right)$. From the remarks preceding the enunciation of this theorem we deduce furthermore that the natural homomorphism of $\operatorname{Ext}\left(Z, E /{ }^{i+1} E\right)$ into $\operatorname{Ext}\left(Z,{ }^{i} E /{ }^{i+1} E\right)$ induces an isomorphism of $\Xi^{*} / \Xi^{* *}$ into $A$.

We note now that ${ }^{i} E={ }^{i} G /{ }_{i} N,{ }^{i+1} E=\left({ }^{i+1} G_{i} N\right) /{ }_{i} N$ and that therefore ${ }^{i} E /{ }^{i+1} E={ }^{i} G /\left({ }^{i+1} G_{i} N\right)$, and this last group is essentially the same as $\left({ }^{i} G /{ }^{i+1} G\right) /\left(\left({ }^{i+1} G_{i} N\right) /{ }^{i+1} G\right)=C / K$ where $C={ }^{i} G /{ }^{i+1} G$ is a free abelian group, since $G$ is a free group $\left.{ }^{35}\right)$. Hence it is possible to represent every abelian extension of $Z$ by ${ }^{i} E /{ }^{i+1} E$ in the form $B=T / D$, where $T$ is the direct product of the free abelian group $C$ by $Z$ and where $1=D \cap Z, K=D Z \cap C$, so that ${ }^{i} E /{ }^{i+1} E, C / K$ and $T / D Z$ are essentially the same group.

$G /{ }^{i+1} G$ is a central extension of the free abelian group $C={ }^{i} G /{ }^{i+1} G$ by $G /{ }^{i} G$. Hence there exists a central factor set $f(x, y)$ of $G /{ }^{i} G$ in $C$ which de-

(35) Magnus [2]. 
termines this central extension. $E /{ }^{i+1} E=G /\left({ }^{i+1} G_{i} N\right)$ is a central extension of ${ }^{i} G /\left({ }^{i+1} G_{i} N\right)=C / K$ by $G /{ }^{i} G$, and this central extension is clearly determined by the central factor set $K f(x, y)$ of $G /{ }^{i} G$ in $C / K$. But $f(x, y)$ is likewise a central factor set of $G /{ }^{i} G$ in the direct product $T$ of $C$ and $Z$, and $D f(x, y)$ is consequently a central factor set of $G /{ }^{i} G$ in $B=T / D$. We denote by $P$ the central extension of $B$ by $G /{ }^{i} G$ which realizes this central factor set $D f(x, y)$.

Since $P$ is a central extension of the abelian group $B$ by the group $G /{ }^{i} G$ of class $i$, it follows that $P$ is of class $i+1$. From $1=D \cap Z$ we infer that $Z$ may be considered a subgroup of $B$ and of $P$. Since $B$ is part of the center of $P$, so is $Z$, and thus $P$ is a central extension of $Z$ by $P / Z$. But $P / Z$ is the central extension of $B / Z$ by $G /{ }^{i} G$ which realizes the central factor set $D Z f(x, y)$ of $G /{ }^{i} G$ in $B / Z=T / D Z$, which latter group is essentially the same as $C / K$ and as ${ }^{i} E /{ }^{i+1} E$. Thus $D Z f(x, y)$ is essentially the same as the central factor set $K f(x, y)$ of $G /{ }^{i} G$ in $C / K$, and we have shown therefore that $P / Z$ and $E /{ }^{i+1} E$ are essentially the same too. Hence $P$ belongs to $\Xi^{*}$. The abelian extension $B$ of $Z$ by ${ }^{i} E /{ }^{i+1} E$ is by its very construction the image of $P$ under the natural homomorphism of $\operatorname{Ext}\left(Z, E /{ }^{i+1} E\right)$ into $\operatorname{Ext}\left(Z,{ }^{i} E /{ }^{i+1} E\right)$. Combining this result with the result obtained in the first paragraph of this proof, we obtain the following somewhat more precise restatement of our theorem.

Corollary 6. The natural homomorphism of $\operatorname{Ext}\left(Z, E /^{i+1} E\right)$ into $\operatorname{Ext}\left(Z,{ }^{i} E /{ }^{i+1} E\right)$ induces an isomorphism of $\Xi^{*} / \Xi^{* *}$ upon $\operatorname{Abext}\left(Z,{ }^{i} E /{ }^{i+1} E\right)$.

The various "natural" homomorphisms occurring in the theorems of this section have been defined in rather different fashions. Still, it is possible to define their product as follows. First we denote by $\alpha^{-1}$ the isomorphism of $\Xi^{*} / \Xi^{* *}$ upon $\operatorname{Abext}\left(Z,{ }^{i} E /{ }^{i+1} E\right)$ which is induced by the natural homomorphism of $\operatorname{Ext}\left(Z, E /{ }^{i+1} E\right)=\Xi$ into $\operatorname{Ext}\left(Z,{ }^{i} E /{ }^{i+1} E\right)$ so that $\alpha$ is a "natural" isomorphism of $\operatorname{Abext}\left(Z,{ }^{i} E /{ }^{i+1} E\right)$ upon $\Xi^{*} / \Xi^{* *}$. Next we denote by $\beta$ the natural homomorphism of $\Xi=\operatorname{Ext}\left(Z, E /{ }^{i+1} E\right)$ into $\Delta=\operatorname{Ext}(Z, E)$, and by $\gamma$ the natural homomorphism of $\Delta$ into $\Sigma=\operatorname{Ext}\left(Z,{ }^{i} E\right)$. These notations will be used throughout.

\section{LEMMA 3. $\Xi^{* * \beta \gamma}=1$.}

Proof. If the central extension $P$ of $Z$ by $E /{ }^{i+1} E$ belongs to $\Xi^{* *}$, then we infer from condition $\left(^{* *}\right)$ that $Z$ is a direct factor of $i P Z$. If $Q$ is the central extension of $Z$ by $E$ upon which $P$ is mapped by $\beta$, then there exist a central factor set $F(X, Y)$ of $E /{ }^{i+1} E$ in $Z$, defining $P$, and a central factor set $f(x, y)$ of $E$ in $Z$, defining $Q$, such that

$$
\left.f(x, y)=F{ }^{i+1} E x,{ }^{i+1} E y\right)
$$

for $x, y$ in $E$.

Since $Z$ is a direct factor of ${ }^{i} P Z$, and since ${ }^{i} E /{ }^{i+1} E=\left({ }^{i} P Z\right) / Z$, we can find elements $t(X)$ in $Z$ for $X$ in ${ }^{i} E /{ }^{i+1} E$ such that $t(1)=1$ and $F(X, Y)$ 
$=t(X) t(Y) t(X Y)^{-1}$ for $X, Y$ in ${ }^{i} E /{ }^{i+1} E$. Let $s(x)=t\left({ }^{i+1} E x\right)$ for $x$ in ${ }^{i} E$. Then we have for $x, y$ in ${ }^{i} E$

$$
\begin{aligned}
f(x, y) & =F\left(^{i+1} E x,{ }^{i+1} E y\right)=t\left({ }^{i+1} E x\right) t\left(^{(i+1} E y\right) t\left(^{i+1} E x y\right)^{-1} \\
& =s(x) s(y) s(x y)^{-1},
\end{aligned}
$$

proving that $Z$ is a direct factor of ${ }^{i} Q Z$, since ${ }^{i} E=\left({ }^{i} Q Z\right) / Z$. The extension $Q$ is therefore mapped by $\gamma$ upon the splitting central extension of $Z$ by ${ }^{i} E$, proving our contention.

From Lemma 3 we infer that $\beta \gamma$ induces a well determined homomorphism $\delta$ of $\Xi^{*} / \Xi^{* *}$ into $\Sigma$, and thus $\alpha \delta$ is a well determined homomorphism of $\operatorname{Abext}\left(Z,{ }^{i} E /{ }^{i+1} E\right)$ into $\Sigma$ which we shall term the natural homomorphism of $\operatorname{Abext}\left(Z,{ }^{i} E /{ }^{i+1} E\right)$ into $\Sigma$. Finally it will be convenient to denote by $\Sigma^{+}$ the image of the group $\Delta^{+}$under the natural homomorphism $\gamma$ so that $\Sigma^{+}$ is a subgroup of $\Sigma$.

Theorem 6. The natural homomorphism of $\operatorname{Abext}\left(Z,{ }^{i} E /{ }^{i+1} E\right)=A$ into $\Sigma$ maps $A$ upon $\Sigma^{+}$, and an element $P$ in $A$ belongs to the kernel of this homomorphism if, and only if $\left.{ }^{36}\right)$, there exists a homomorphism of ${ }^{i} E /\left({ }^{i} E\right.$, $\left.{ }^{i} E\right)$ into $P$ which induces the identity in ${ }^{i} E /{ }^{i+1} E$.

Proof. It is a consequence of Corollary 6 that $A$ is mapped by the natural isomorphism $\alpha$ upon the group $\Xi^{*} / \Xi^{* *}$. It is a consequence of the definition of $\Sigma^{+}$that $\Delta^{+}$is mapped by the natural homomorphism $\gamma$ upon the group $\Sigma^{+}$, and we infer from Theorem 4 that the natural homomorphism $\beta$ induces an isomorphism of $\Xi^{*}$ upon $\Delta^{+}$. Thus $\beta \gamma$ induces a homomorphism $\delta$ of $\Xi^{*} / \Xi^{* *}$ upon $\Sigma^{+}$, and $\alpha \delta$ is a homomorphism of $A$ upon $\Sigma^{+}$.

Denote by $K$ the kernel of the homomorphism $\alpha \delta$. Then $K^{\alpha}$ is a subgroup $M / \Xi^{* *}$ of $\Xi^{*} / \Xi^{* *}$, and $\alpha$ and $\beta$ being isomorphisms in the groups under consideration, it follows that $M^{\beta}=B$ is the cross cut of the kernel of $\gamma$ and of $\Delta^{+}$, and thus $B$ is the kernel of the homomorphism of $\Delta^{+}$upon $\Sigma^{+}$which is induced by $\gamma$.

Since $\gamma$ is the natural homomorphism of $\operatorname{Ext}(Z, E)$ into $\operatorname{Ext}\left(Z,{ }^{i} E\right)$, and since ${ }^{i} E=\left({ }^{i} W Z\right) / Z$ for $W$ in $\operatorname{Ext}(Z, E)$, it follows that $W$ in $\operatorname{Ext}(Z, E)$ belongs to the kernel of $\gamma$ if, and only if, $Z$ is a direct factor of ${ }^{i} W Z$.

Suppose now that $P$ belongs to $\operatorname{Abext}\left(Z,{ }^{i} E /{ }^{i+1} E\right)$. It is a consequence of the definition of the natural isomorphism $\alpha$ that $P^{\alpha}$ is a coset of $\Xi^{*} / \Xi^{* *}$. If $Q$ is any element in the $\operatorname{coset} P^{\alpha}$, then $Q$ is mapped upon $P$ by the natural homomorphism of $\operatorname{Ext}\left(Z, E /{ }^{i+1} E\right)$ into $\operatorname{Ext}\left(Z,{ }^{i} E /{ }^{i+1} E\right)$. Consequently we may consider $P$ as the uniquely determined subgroup of $Q$, satisfying:

$$
Z \leqq P \text { and } P / Z={ }^{i} E /{ }^{i+1} E \text { or } P=Z^{i} Q \text {. }
$$

$Q$ is a central extension of $Z$ by $E /{ }^{i+1} E$, and $Q$ is therefore determined

(36) Compare this condition with (ii) of Theorem 2. 
by a central factor set $F(X, Y)$ of $E /{ }^{i+1} E$ in $Z$. The natural homomorphism $\beta$ of $\operatorname{Ext}\left(Z, E /{ }^{i+1} E\right)$ into $\operatorname{Ext}(Z, E)$ maps, by its definition, the extension $Q$ upon the central extension $R$ of $Z$ by $E$ which is determined by the central factor set $f(x, y)$, defined by the rule:

$$
f(x, y)=F\left({ }^{i+1} E x,{ }^{i+1} E y\right) \quad \text { for } x, y \text { in } E .
$$

Finally it should be remembered that $R$ is mapped by the natural homonorphism $\gamma$ of $\operatorname{Ext}(Z, E)$ in to $\operatorname{Ext}\left(Z,{ }^{i} E\right)$ upon the uniquely determined subgroup $S$ of $R$, satisfying $Z \leqq S \leqq R$ and $S / Z={ }^{i} E$; or $S=Z^{i} R$.

A. Suppose that $P$ belongs to the kernel $K$ of the natural homomorphism $\alpha \delta$. Then $S$ is the splitting extension of $Z$ by ${ }^{i} E$, and there exists therefore a single-valued ${ }^{i} E$ to $Z$ function $t(x)$ such that $t(1)=1$ and $f(x, y)=t(x) t(y) t(x y)^{-1}$ for $x, y$ in $i E$. If $x$ happens to be in ${ }^{i+1} E$, then we infer from the definition of $f(r, s)$ that $1=F\left(1,{ }^{i+1} E y\right)=f(x, y)=t(x) t(y) t(x y)^{-1}$, and since a like argument applies, if $y$ is in ${ }^{i+1} E$, we have shown:

(a) $t(x y)=t(x) t(y)$, if $x$ or $y$ is in ${ }^{i+1} E$.

Since the elements $i(z)$ belong to the abelian group $Z$, we infer $t(x y)=t(y x)$ from (a) whenever $x$ is in ${ }^{i} E$ and $y$ is in ${ }^{i+1} E$. This implies in particular:

(b) $t(x)=1$ for $x$ in $\left({ }^{i} E,{ }^{i+1} E\right)$. Combining (a) and (b) we obtain:

(c) $t(x)$ induces a homomorphism of ${ }^{i+1} E /\left({ }^{i} E,{ }^{i+1} E\right)$ into $Z$.

If $X$ is a coset of ${ }^{i} E /{ }^{i+1} E$, then let $r(X)$ be a representative of this coset, $r(1)=1$. Put $s(X)=t(r(X))^{-1}$. Now we deduce from (a) and the commutativity of $P:$ If $X$ and $Y$ are in ${ }^{i} E /{ }^{i+1} E$, then

$F(X, Y) s(X) s(Y) s(X Y)^{-1}$

$$
\begin{aligned}
& =f(r(X), r(Y)) s(X) s(Y) s(X Y)^{-1} \\
& =t(r(X)) t(r(Y)) t(r(X) r(Y))^{-1} t(r(X))^{-1} t(r(Y))^{-1} t(r(X Y)) \\
& =t(r(X) r(Y))^{-1} t(r(X Y))=t\left(r(X) r(Y) r(X Y)^{-1}\right)^{-1},
\end{aligned}
$$

since $r(X) r(Y) r(X Y)^{-1}$ belongs to ${ }^{i+1} E$. The elements $\left({ }^{i} E,{ }^{i+1} E\right) r(X) r(Y) r(X Y)^{-1}$ form a central factor set of ${ }^{i} E /{ }^{i+1} E$ in ${ }^{i+1} E /\left({ }^{i} E,{ }^{i+1} E\right)$, defining ${ }^{i} E /\left({ }^{i} E,{ }^{i+1} E\right)$, and this central factor set is mapped by the homomorphism $t(v)^{-1}$ of ${ }^{i+1} E /\left({ }^{i} E,{ }^{i+1} E\right)$ into $Z$-by (c) - upon a central factor set associated to $F(X, Y)$. Hence there exists a homomorphism $\eta$ of ${ }^{i} E /\left({ }^{i} E,{ }^{i+1} E\right)$ into $P$ which induces the identity in ${ }^{i} E /{ }^{i+1} E$ and which induces $t(v)^{-1}$ in ${ }^{i+1} E /\left({ }^{i} E,{ }^{i+1} E\right)$. But $P$ is abelian, and thus $\left({ }^{i} E,{ }^{i} E\right)$ is mapped upon 1 by $\eta$. Since $\left({ }^{i} E,{ }^{i+1} E\right)$ $\leqq\left(i E,{ }^{i} E\right) \leqq{ }^{i+1} E$, we have shown the existence of a homomorphism of ${ }^{i} E /\left({ }^{i} E,{ }^{i} E\right)$ into $P$ which induces the identity in ${ }^{i} E /{ }^{i+1} E$, and we have proved the necessity of our condition.

B. Suppose now that there exists a homomorphism of ${ }^{i} E /\left({ }^{i} E,{ }^{i} E\right)$ into $P$ which induces the identity in ${ }^{i} E /{ }^{i+1} E$. Then there exists clearly a homomorphism $\nu$ of ${ }^{i} E$ into $P$ which induces the identity in ${ }^{i} E /{ }^{i+1} E$. In every 
coset $X$ of ${ }^{i} E /{ }^{i+1} E$ select a representative $r(X)$; in particular $r(1)=1$. The element $r(X)^{v}$ is then a representative of the coset of $P / Z$ which corresponds to $X$ in ${ }^{i} E{ }^{i+1} E$, and the elements $r(X)^{\nu} r(Y)^{\nu} r(X Y)^{-\nu}$ form therefore a central factor set of ${ }^{i} E /{ }^{i+1} E$, defining the abelian extension $P$ of $Z$ by ${ }^{i} E /{ }^{i+1} E$. Consequently there exist elements $c(X)$ in $Z$ such that $c(1)=1$ and $r(X)^{r} r(Y)^{r} r(X Y)^{-v}=c(X) c(Y) c(X Y)^{-1} F(X, Y)$ for $X, Y$ in ${ }^{i} E /^{i+1} E$.

If $x$ is an element in ${ }^{i} E$, then $x^{-1} r\left({ }^{i+1} E x\right)$ belongs to ${ }^{i+1} E$. Hence $s(x)$ $=c\left({ }^{i+1} E x\right)^{-1}\left(x^{-1} r\left({ }^{i+1} E x\right)\right)$ is, for every $x$ in ${ }^{i} E$, an element in $Z$; in particular $s(1)=1$. Since $P$ is an abelian group, we find

$$
\begin{aligned}
s(x) s(y) s(x y)^{-1} & =c\left({ }^{i+1} E x\right)^{-1} c\left({ }^{i+1} E y\right)^{-1} c\left({ }^{i+1} E x y\right) r\left({ }^{i+1} E x\right)^{\nu} r\left({ }^{i+1} E y\right)^{\nu} r\left({ }^{i+1} E x y\right)^{-\nu} \\
& =F\left(^{i+1} E x,{ }^{i+1} E y\right)=f(x, y) .
\end{aligned}
$$

Thus we have shown that the defining central factor set of the central extension $S$ of $Z$ by ${ }^{i} E$ is a transformation set. But $S$ is the map of $P$ under the natural homomorphism of $\operatorname{Abext}\left(Z,{ }^{i} E /{ }^{i+1} E\right)$ upon $\Sigma^{+}$, and thus we have shown that $P$ belongs to the kernel $K$, proving the sufficiency of our condition.

Corollary 7. If ${ }^{i} E /\left({ }^{i} E,{ }^{i} E\right)$ is a splitting extension of ${ }^{i+1} E /\left({ }^{i} E,{ }^{i} E\right)$ by ${ }^{i} E /{ }^{i+1} E$, then the natural homomorphism of $\operatorname{Abext}\left(Z,{ }^{i} E /{ }^{i+1} E\right)$ upon $\Sigma^{+} i$ is an isomorphism.

Proof. There exists a subgroup $V$ of ${ }^{i} E /\left({ }^{i} E,{ }^{i} E\right)$ such that ${ }^{i} E /\left({ }^{i} E,{ }^{i} E\right)$ $=V\left[{ }^{i+1} E /\left({ }^{i} E,{ }^{i} E\right)\right]$ and $1=V \cap\left[{ }^{i+1} E /\left({ }^{i} E,{ }^{i} E\right)\right]$. If $P$ is an abelian extension of $Z$ by ${ }^{i} E /{ }^{i+1} E$, and if the homomorphism $\eta$ of ${ }^{i} E /\left({ }^{i} E,{ }^{i} E\right)$ into $P$ induces the identity in ${ }^{i} E /{ }^{i+1} E$, then $\eta$ effects an isomorphism of $V$, and every coset of $P / Z$ contains one and only one element of $V^{\eta}$, proving that $P$ is a splitting abelian extension of $Z$ by ${ }^{i} E /{ }^{i+1} E$. Now our contention is an immediate consequence of Theorem 6 .

THEOREM 7. The system of the ith representation groups of $G / N$ is exactly a coset of $\Sigma / \Sigma^{+}$.

Proof. If $L$ is an $i$ th representation group of $G / N$, then $L$ is essentially the same as a group ${ }^{i} G / W$ where ${ }_{i+1} N \leqq W$ and where ${ }_{i} N /{ }_{i+1} N$ is the direct product of $W /{ }_{i+1} N$ and of $\left({ }_{i} N \cap{ }^{i+1} G\right) /{ }_{i+1} N$. Thus $G / W$ is a central extension of $Z$ by $E$, and there exists a homomorphism $\eta$ of $H=G /{ }_{i+1} N$ upon $G / W$ which maps $M={ }_{i} N /{ }_{i+1} N$ upon $Z=\left({ }_{i} N \cap{ }^{i+1} G\right) /{ }_{i+1} N$ and which leaves invariant every element in $E$ and in $Z$. Consequently $G / W$ belongs to the system $\Delta^{\circ}$, and this system is by Corollary 5 a coset of $\Delta / \Delta^{+}$. It is furthermore clear that every group in $\Delta^{\circ}$ may be represented in this form $G / W$. But the $i$ th representation groups of $G / N$ are just the images of these groups $G / W$, that is, of the groups in $\Delta^{\circ}$, under the natural homomorphism $\gamma$ of $\Delta$ into $\Sigma$. Since $\Delta^{+}$is mapped by $\gamma$ upon $\Sigma^{+}$, it follows now that the system of the $i$ th representation groups of $G / N$ is exactly the coset $\left[\Delta^{\circ}\right]^{\gamma}$ of $\Sigma / \Sigma^{+}$, as we intended to show. 
Corollary 8. If ${ }^{i} G /\left[\left({ }^{i} G,{ }^{i} G\right)_{i} N\right]$ is a splitting extension of $\left[{ }^{i+1} G_{i} N\right] /\left[\left({ }^{i} G,{ }^{i} G\right){ }_{i} N\right]$ by ${ }^{i} G /\left({ }^{i+1} G{ }_{i} N\right)$, then the system of the ith representation groups of $G / N$ is a coset of the group $\operatorname{Ext}\left[\left({ }_{i} N \cap{ }^{i+1} G\right) /{ }_{i+1} N,{ }^{i} G /{ }_{i} N\right]$ modulo a subgroup which is a natural isomorphic map of Abext $\left[\left({ }_{i} N \cap{ }^{+1} G\right) /{ }_{i+1} N,{ }^{i} G /\left({ }^{i+1} G G_{i} N\right)\right]$.

This is an almost immediate consequence of Theorem 7 and of Corollary 7 , if one substitutes-as we did in the proof of Theorem 7-for $E, Z$ and $\Sigma$ their original definitions.

Remark 3. It is clear that the condition of Corollary 8 is satisfied whenever ${ }^{i+1} G_{i} N=\left({ }^{i} G,{ }^{i} G\right){ }_{i} N$. This is obviously the case for $i=0$. It has been pointed out in Remark 1 of $\S 3$ that $\left(N \cap^{1} G\right) /{ }_{1} N$ is just the multiplicator of $G / N$. Corollary 8 reduces, therefore, for $i=0$ to a result that-at least for finite $G / N$-has already been obtained by I. Schur and others $\left({ }^{37}\right)$.

COROLlary 9. If every abelian extension of $\left({ }_{i} N \cap{ }^{i+1} G\right) /{ }_{i+1} N$ by ${ }^{i} G /\left({ }^{i+1} G_{i} N\right)$ is a splitting extension, then there exists one and only one ith representation group of $G / N$.

Proof. It follows from the hypothesis of this corollary that $\operatorname{Abext}\left(Z,{ }^{i} E /{ }^{i+1} E\right)$ $=1$. Thus the contention of this corollary is an immediate consequence of Theorems 7 and 6.

Remark 4. The hypothesis of Corollary 9 is certainly satisfied whenever ${ }^{i} G /\left({ }^{i+1} G_{i} N\right)$ is a free abelian group. It is furthermore satisfied whenever $\left({ }_{i} N \cap{ }^{i+1} G\right) /{ }_{i+1} N$ and ${ }^{i} G /\left({ }^{i+1} G_{i} N\right)$ are finite abelian groups of relatively prime order.

Corollary 10. If $N \leqq(G, G)$, then there exists, for every $i$, one and only one ith representation group of $G / N$.

Proof. From $N \leqq(G, G)$ one deduces by complete induction that ${ }_{i} N \leqq{ }^{i+1} G$. Hence ${ }^{i} G /\left({ }^{i+1} G_{i} N\right)={ }^{i} G /{ }^{i+1} G$, and this group is a free abelian group, since $G$ is a free group $\left.{ }^{38}\right)$. Now Corollary 10 is an immediate consequence of Corollary 9.

Remark 5. It is easy to see that our main results like Theorem 7 and Corollary 8 would not be true without the hypothesis that $G$ is a free group. Explicit use has been made of this hypothesis in only three instances, namely in the proofs of Corollaries 3 and 5 and of Theorem 5.

Keizo Asana-Kenjiro Shoda

\section{BIBLIOGRAPHY}

1. Zur Theorie der Darstellungen einer endlichen Gruppe durch Kollineationen, Compositio Math. vol. 2 (1935) pp. 230-240.

R. BAER

1. The subgroup of the elements of finite order of an abelian group, Ann. of Math. vol. 37 (1936) pp. 766-781.

(37) See footnote 11 above.

(38) Magnus [2]. 
2. Abelian groups without elements of finite order, Duke Math. J. vol. 3 (1937) pp. 68-122.

3. Groups with abelian central quotient. group, Trans. Amer. Math. Soc. vol. 44 (1938) pp. 357-386.

4. The higher commutator subgroups of a group, Bull. Amer. Math. Soc. vol. 50 (1944) pp. 143-160.

5. Groups without proper isomorphic quotient groups, Bull. Amer. Math. Soc. vol. 50 (1944) pp. 267-278.

A. H. Clifford and S. Maclane

1. Factor sets of a group in its abstract unit group, Trans. Amer. Math. Soc. vol. 50 (1941) pp. 385-406.

S. EILENBERg AND MACLANE

1. Relations between homotopy and homology groups, Proc. Nat. Acad. Sci. U.S.A. vol. 29 (1943) pp. 155-158.

2. Relations between homology and homotopy groups in spaces, Ann. of Math. vol. 46 (1945) P. Hall pp. 450-509.

1. A contribution to the theory of groups of prime power order, Proc. London Math. Soc. vol. 36 (1934) pp. 29-95.

H. HopF

1. Fundamentalgruppe und zweite Bettische Gruppe, Comment, Math. Helv. vol. 14 (1942) pp. 257-309.

\section{S. LEFSCHETZ}

1. Algebraic topology, Amer. Math. Soc. Colloquium Publications, vol. 27, 1942.

W. Magnus

1. Beziehungen zwischen Gruppen und Idealen in einem speziellen Ring, Math. Ann. vol. 111 (1935) pp. 259-280.

2. Über freie Faktorgruppen und freie Untergruppen gegebener Gruppen, Monatshefte für Mathematik und Physik vol. 47 (1938) pp. 307-313.

G. A. Miller

1. On the representation group of a given abstract group, Trans. Amer. Math. Soc. vol. 14 (1913) pp. 444-452.

2. Representation groups of well known systems of groups, Tôhoku Math. J. vol. 6 (1914) pp. 35-41.

B. H: NEUMANN

\section{SCHUR}

1. Identical relations in groups. I, Math. Ann. vol. 114 (1937) pp. 506-525.

1. Über die Darstellungen der endlichen Gruppen durch gebrochene lineare Substitutionen, J. Reine Angew. Math. vol. 127 (1904) pp. 20-50.

2. Untersuchungen ibber die Darstellungen der endlichen Gruppen durch gebrochene lineare Substitutionen, J. Reine Angew. Math. vol. 132 (1907) pp. 85-137.

H. Zassenhaus

1. Lehrbuch der Gruppentheorie. I, Hamburger Mathema tische Einzelschriften, vol. 21, 1937.

UNIVERSITY OF ILLINOIS,

URBANA, ILL. 